\title{
Shaping the Collision Selectivity in a Looming Sensitive Neuron Model with Parallel ON and OFF Pathways and Spike Frequency Adaptation
}

\author{
Qinbing $\mathrm{Fu}^{\mathrm{a}}$, Cheng $\mathrm{Hu}^{\mathrm{a}}$, Jigen Peng ${ }^{\mathrm{b}}$, Shigang Yue ${ }^{\mathrm{a}, *}$ \\ ${ }^{a}$ Computational Intelligence Laboratory (CIL), University of Lincoln, Lincoln, UK \\ ${ }^{b}$ School of Mathematics and Statistics, Xi'an Jiaotong University, Xi'an, China
}

\begin{abstract}
Shaping the collision selectivity in vision-based artificial collision-detecting systems is still an open challenge. This paper presents a novel neuron model of a locust looming detector, i.e. the lobula giant movement detector (LGMD1), in order to provide effective solutions to enhance the collision selectivity of looming objects over other visual challenges. We propose an approach to model the biologically plausible mechanisms of ON and OFF pathways and a biophysical mechanism of spike frequency adaptation (SFA) in the proposed LGMD1 visual neural network. The ON and OFF pathways can separate both dark and light looming features for parallel spatiotemporal computations. This works effectively on perceiving a potential collision from dark or light objects that approach; such a bio-plausible structure can also separate LGMD1's collision selectivity to its neighbouring looming detector - the LGMD2. The SFA mechanism can enhance the LGMD1's collision selectivity to approaching objects rather than receding and translating stimuli, which is a significant improvement compared with similar LGMD1 neuron models. The proposed framework has been tested using off-line tests of synthetic and real-world stimuli, as well as online bio-robotic tests. The enhanced collision selectivity of the proposed model has been validated in systematic experiments. The computational simplicity and
\end{abstract}

\footnotetext{
* Corresponding author

Email addresses: qifu@lincoln.ac.uk (Qinbing Fu), chu@lincoln.ac.uk (Cheng Hu), jgpeng@mail.xjtu.edu.cn (Jigen Peng), syue@lincoln.ac.uk (Shigang Yue)
} 
robustness of this work have also been verified by the bio-robotic tests, which demonstrates potential in building neuromorphic sensors for collision detection in both a fast and reliable manner.

Keywords: locusts, LGMD1, neuron model, collision detection, collision selectivity, ON and OFF pathways, spike frequency adaptation, bio-robotics

\section{Introduction}

Collision detection is of critical importance for mobile machines, like ground vehicles, UAVs and robots. Although there are plenty of physical sensors used for collision detection, such as infra-red, radar, laser, ultrasound, vision and

5 various combinations of these, it is still an open challenge for mobile machines to detect a collision in both a timely and robust manner, without human intervention, especially in complex and dynamic environments. Vision, amongst these sensing modalities, can extract useful motion cues from dynamic scenes.

For real-time collision detection, there are many conventional computer vision strategies. The vast majority of methods implement object and scene segmentation, estimation or classification algorithms [1, 2. Some vision-based collision-detecting systems have also been applied in ground-vehicles handling driving scenarios to improve road safety [1]. In addition, the state-of-the-art visual sensors like RGB-D [3], Kinect [4, 5] and event-driven cameras [6, can provide mobile machines with more abundant visual features compared to traditional cameras which facilitate obstacle recognition, object segmentation and map construction for collision detection. However, these vision-based techniques, based on segmentation, classification and localisation algorithms, are either computationally costly or heavily relying upon specific visual sensors. In addition, the efficiency of these approaches also depends on the degree of complexity required in real-world physical scenes. As a result, a fast, reliable and low-power method for collision detection in complex and dynamic scenes is required for future intelligent machines.

Millions of years of evolutionary development has produced, in nature, ani- 


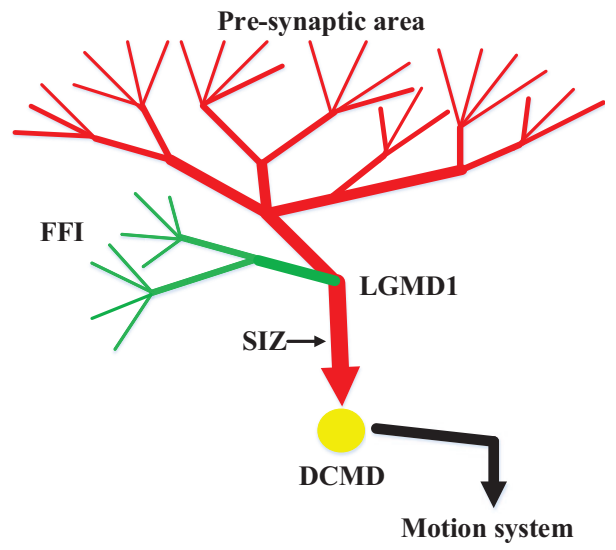

Figure 1: Schematic illustration of the morphological LGMD1 neural network: the red 'dendrites' field indicates pre-synaptic visual processing with the proposed ON and OFF mechanisms; the green 'dendrites' field denotes a separate feed-forward inhibitory (FFI) pathway to the LGMD1; SIZ is short for the 'spike initiation zone' for the proposed spike frequency adaptation mechanism. DCMD (descending contralateral motion detector) is a one-to-one post-synaptic target neuron conveying spikes to further motion control neural systems.

mals that possess robust and efficient vision systems capable of collision perception to deal with a variety of aspects of life, including foraging, escaping from predators, chasing mates and so forth. Insects, in particular, have a relatively small number of visual neurons compared with vertebrates and humans but can still navigate smartly through visually cluttered and dynamic environments 7 .

30 Understanding insects' collision perception strategies are not only attractive to neural system modellers, but also critical in providing effective solutions for future robots [8, 7, 9]. More specifically, both locusts [10, 11, 12, 13] and flies [14 possess the amazing ability to perceive impending collisions in complex and dynamic scenes. These biological visual systems provide inspiration to computational modellers to build neuromorphic collision detectors in mobile robotic applications [15, 16, 17, 18, 19, 20, 21, ground vehicles [22, 23, 24, 25] and UAVs [26, 7.

Amongst these insect looming detectors, the lobula giant movement detectors (LGMDs) in the locusts' optic lobe is known to respond most strongly 
to direct and fast approaching objects [10, 11, 13. Biologists have explored two neighbouring LGMDs, i.e. LGMD1 and LGMD2, and moreover revealed the different collision selectivity between them: the LGMD2 is only sensitive to dark looming objects, whilst the LGMD1 can detect either dark or light approaching stimuli [10, 11, 13]. The LGMD1 (Fig. 1), in particularly, has

45 generated many simple solutions for collision detection in mobile machines, e.g. [27, 16, 17, 18, 19. However, shaping the collision selectivity to looming objects in these collision perception neural systems, is a big challenge to computational modellers, as other kinds of visual stimuli like the translation and the recession, significantly affect the accuracy of collision detections. In this study, we propose a method to enhance the collision selectivity with two biologically plausible structures and mechanisms - the ON and OFF pathways and the spike frequency adaptation.

The ON and OFF pathways have been explored in the visual systems of many animals such as flies [28, 29, 30, 31, 32, 33] and vertebrates [34; these 55 include mammals like rabbits [35], mice [36], cats [37] and monkeys [38. Such a structure reveals an important theory of encoding visual information separately in an animal's preliminary motion-detecting circuitry: luminance increments and decrements flow into the ON and OFF pathways, respectively [30, 35, 31, [36]. The underlying functionality of $\mathrm{ON}$ and $\mathrm{OFF}$ pathways has generated 60 many biological motion detectors $39,40,41,42,28$. It has also been applied to computational models of direction-selective neurons [43] and small target movement detectors [44,45] in the fly's preliminary visual systems. Our previous partial studies have demonstrated the potential of such polarity pathways in modelling LGMD2 looming detectors [20, 21. In this paper, we apply the bioplausible ON and OFF pathways to model the LGMD1 looming detector and more importantly investigate its underlying functionality and characteristics.

The biophysical mechanism of spike frequency adaptation has been revealed to play an important role in shaping the collision selectivity of LGMD1 for looming stimuli versus translation and recession in previous biological research 70 46, 47. In this paper, we continue on demonstrating its potential computational 
role and furthermore the efficacy in real-world collision detection tasks. Our systematic experiments ranging from off-line synthetic and real-world stimuli tests to on-line bio-robotic tests demonstrate the following contributions of this neuron modelling study:

- The functionality of proposed ON and OFF pathways enable us to separate the collision selectivity of LGMD1 to its neighbouring looming-sensitive neuron - the LGMD2, which has specific collision selectivity to dark looming objects [11, 20, 21]. Such a structure can fully realise the underlying characteristics of a biological LGMD1 neuron in locusts, which works effectively on collision recognition of both light and dark objects that approach.

- The computational mechanism of spike frequency adaptation significantly enhances the collision selectivity of LGMD1 in real-world tests which is an advance over similar LGMD1 neuron models.

- The proposed method yields simple solutions for collision perception in both an efficient and reliable manner, that only requires a monocular camera and fewer computational resources than conventional computer vision methods. This has been verified by our bio-robotic tests.

- The proposed neuron model evidences that the ON and OFF pathways play roles in the locusts' visual systems, though little physiological and anatomical evidence has been found to date.

In the following sections, we review some related works in Section 2 , The proposed LGMD1 neuron model framework is presented in Section 3 . Experimental evaluation along with results and analysis is provided in Section 4 . Further discussion is given in Section 5 Finally, we conclude this work in Section 95 6.

\section{Related work}

Within this section, some relevant works to the proposed LGMD1 neuron model will be reviewed, specifically in the areas relating to biologically inspired 
collision-detecting systems, biologically plausible ON and OFF pathways and optical flow problem [7.

To address these optical flow based problems using only a vision-based sensor, modellers can take inspiration from locusts. Unlike flies, locusts do not have 
similar LPTCs for an optical flow-based strategy, yet they have specific neurons that sense looming stimuli, the LGMDs, that are caused by incoming objects. These respond most strongly to direct-collision from onward coming objects, a situation that the optical-flow neurons cannot deal with very well.

Locust inspired collision detectors. The LGMDs looming detectors are crucial for the survival of locusts, from adolescence to adulthood, for perceiving imminent collision corresponding to various avoidance behaviours such as hiding, jumping and sliding during flight [54, 55. The collision selectivity in these locust looming detectors has been solidly formed, representing the highest firing rates by approaching objects amongst other visual stimuli.

Inspired by such fascinating visual neurons, there are a few collision-free neural vision systems that have been designed, and successfully applied in applications, not only for ground vehicles [23, 24, 22, but also vision-based robots [18, 15, 56, 19, 27, 17, 16. These works have demonstrated the effectiveness and robustness of LGMDs-based models in collision detection, however, shaping the collision selectivity to looming objects only, is still an open challenge to computational modellers, as these collision detectors are easily affected by irrelevant movements like translational and recessive motion, especially in complex and dynamic environments. To improve the collision selectivity of these looming detectors, some solutions have been proposed either to monitor the gradient change of LGMD1 neural responses [57, or to combine the functionality of LGMD1 model with a translating sensitive neural network [25. In addition, Badia et al. proposed a seminal work of a non-linear LGMD1 model which can discriminate approaching from receding stimuli well [16].

Compared with above methods, we propose an alternative way to shape the collision selectivity with biologically plausible pathways and mechanisms, which demonstrates the following advantages over previous LGMD1 models:

- In comparison to the non-linear LGMD1 model proposed in [16, we apply linear spatiotemporal computations in the pre-synaptic field of LGMD1 neural network, which requires fewer computational resources. Moreover, 
the $\mathrm{ON}$ and $\mathrm{OFF}$ pathways in the proposed neuron model can achieve previous research.

- Compared with the linear LGMDs models [27, 15, 20, 21], the computational modelling of spike frequency adaptation can suggest a simpler solution to mediate the LGMD1's selectivity to approaching rather than translating and receding stimuli.

\section{2. $O N$ and $O F F$ visual pathways}

In the proposed neuron model, we highlight the functionality of $\mathrm{ON}$ and $\mathrm{OFF}$ visual pathways. As mentioned in Section 1 little evidence has been found that the ON and OFF pathways exist in a locust's visual system [58, 59. While the biological substrate has not been fully identified by biologists, the computational modelling studies and bio-robotic solutions are particularly useful to provide new biological hypotheses, as the models and robots can be tested in experimental conditions similar to physiological and ethological experiments.

In the vast majority of LGMD1 neuronal models, visual information is processed in a single pathway, as shown in Fig. 2a. Compared with the seminal work on modelling ON and OFF mechanisms in an LGMD-based computational model by Keil et al. [60, we model the ON and OFF pathways each with multiple layers for spatiotemporal computations and investigate its functionality of achieving different collision selectivity. In addition, the nonlinear modelling study proposed in [16], also demonstrated the effectiveness of an ON and OFF mechanism of encoding for onset and offset responses separately to implement a biological LGMD1 neuron. More specifically, luminance increments/decrements give rise to onset and offset responses, respectively. With a similar idea in the proposed LGMD1 neuron model, we demonstrate that the onset and offset responses bring about delayed inhibitory and excitatory information in the ON and OFF pathways respectively, as can be seen in the schematic diagram shown in Fig. 2c. While in previous LGMD1 models like 2a, the inhibitory flows are always delayed relative to the excitatory flows. Moreover, as shown in Fig. 2b. 


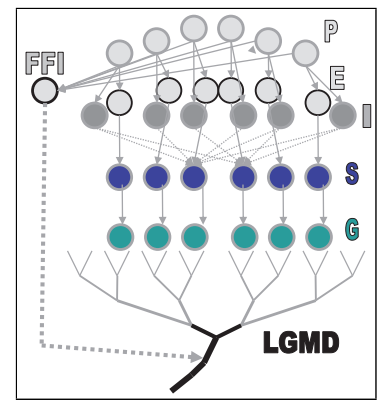

(a) comparative LGMD1

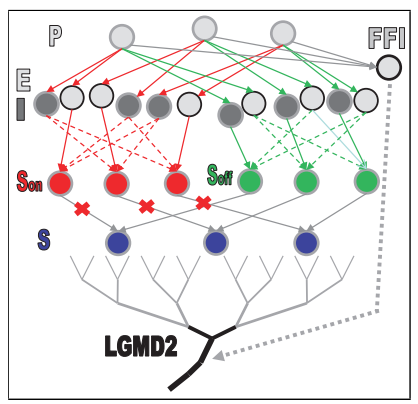

(b) previous LGMD2

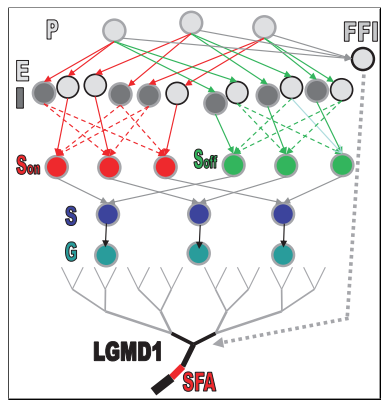

(c) proposed LGMD1

Figure 2: Schematics of former LGMDs models adapted from [27, 15, 21, 20] and the proposed LGMD1 model: (a) The previous LGMD1 model [27, 15] (taken 6 pixels from the visual field) processes visual information in a single pathway, that is composed of five layers (P, E, I, S, G) and two cells (FFI, LGMD). (b) The LGMD2 model from our previous research 21, 20] (taken 3 pixels) processes signals in separated ON (red-arrows) and OFF (green-arrows) pathways each with three layers (E, I, S), whilst the ON channels are rigorously blocked. (c) The proposed LGMD1 model processes signals in the ON and OFF pathways without bias, whilst a new SFA mechanism is modelled. In all models, the dashed lines indicate transmissions of delayed neural signals.

we recently demonstrated the potential and usefulness of ON and OFF pathways in the modelling of a biological LGMD2 neuron [20, 21].

\subsection{Spike frequency adaptation}

Another important biological theory guiding this modelling study is a biophysical mechanism of spike frequency adaptation. Compared with similar LGMDs-based models, such as [27, 15, 21, 20] in Fig. 2, the SFA mechanism is for the first time modelled in the LGMD1 visual neural network. Generally speaking, it explains the fundamental phenomenon of an 'adaptive status' in neural processing of auditory, visual and other sensory systems. Specifically for LGMD1, the biological studies demonstrate that such an adaptive mechanism contributes significantly to mediate the collision selectivity of approaching, over receding and translating objects [61, 46, 47]. There are a few assumptions of its computational roles [4], one of which is reconciled with a high-pass filtering in the spike initiation zone (SIZ in Fig. 1). 
For biological looming detectors in locusts, when challenged by the translational movements at a constant speed, a fixed number of photoreceptors in the

field of view are activated, which makes the neurons liable to adaptation, i.e., the neural response decays quickly [61, 46]. In addition, the receding stimuli give rise to a reducing number of activated photoreceptors, which also leads to adaptation [46, 47]. On the other hand, in the case of looming, an increasing number of photoreceptors will be activated, a situation in which the neurons likely overwhelm adaptation and represent high firing rates [61, 46].

\section{Framework of the proposed neuron model}

In this section, we present the proposed LGMD1 neuron model. In the pre-synaptic field to the LGMD1 cell, compared with similar LGMD1 works [27, 15], we highlight the functions of separated ON and OFF pathways with spatial convolution and dynamically temporal filtering. A new mechanism of spike frequency adaptation is modelled in the spike initiation zone. Generally speaking, as depicted in Fig 3 the LGMD1 model includes (1) a photoreceptors layer to retrieve initial motion information, (2) two separated visual pathways to encode ON and OFF depth features - each of which has three local layers of excitation, inhibition and summation cells, (3) a summation-grouping layer to combine relayed excitations from both pathways, (4) a LGMD1 cell to exponentially map feed-forward excitation to membrane potential, (5) an individual feed-forward pathway for an 'all-or-none' law to control the activation of LGMD1, (6) SFA and Spiking mechanisms to transform neural response to spikes.

It is also important to state that the proposed framework only involves lowlevel image processing strategies and perceiving collisions by reacting to the expanding edges. Fig. 3 depicts a schema of the proposed framework with underlined PNN structure and spatiotemporal convolutions. Table 1 illustrates the abbreviations of components in this visual neural network. 


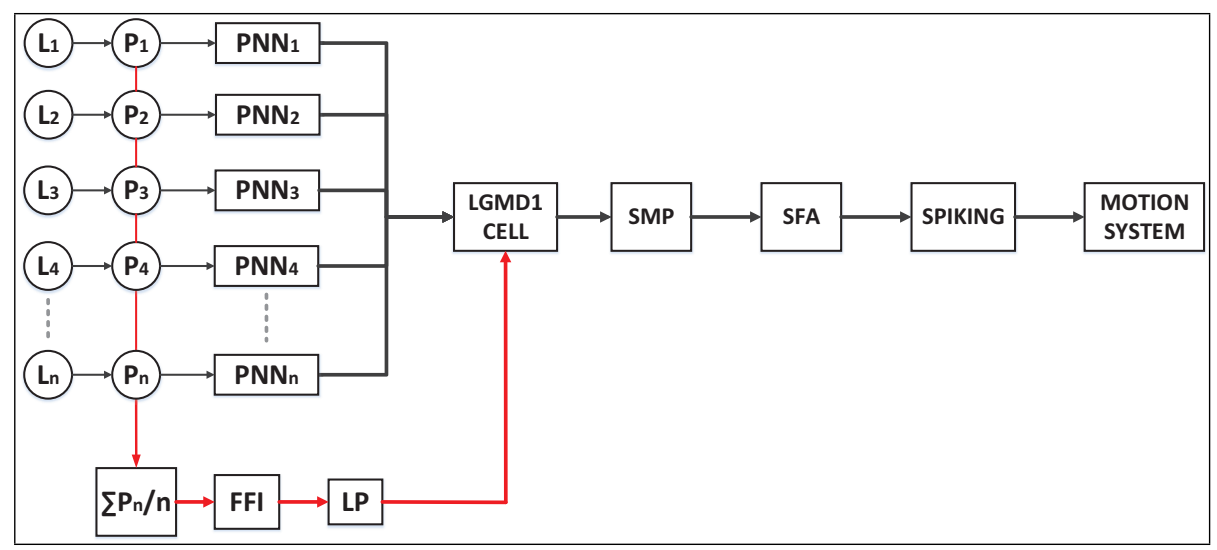

(a) LGMD1 visual neural network

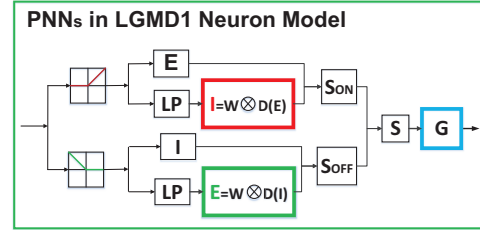

(b) partial neural network

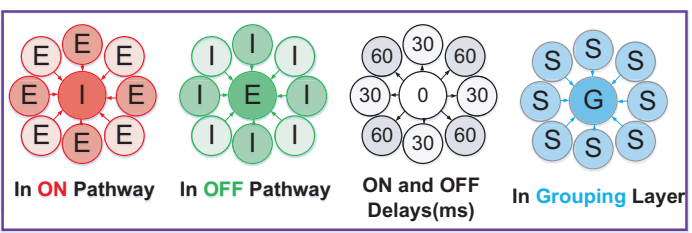

(c) spatiotemporal convolutions

Figure 3: Schematic of LGMD1 neural network for collision detection. (a) A schema of signal processing in LGMD1 model: the pixel-wise luminance $(\mathrm{L})$ is captured by photoreceptors (P), which convey motion information to the partial neural networks (PNN); the LGMD1 cell integrates the local excitations from intact pre-synaptic PNNs forming the sigmoid membrane potential (SMP) towards the spike frequency adaptation (SFA) and spiking mechanisms; the generated spikes are transmitted to motion neural systems. (b) A schema of PNN depicts the ON and OFF mechanisms: in ON channels, the inhibition (I) is computed via convolving surrounding delayed excitations $(\mathrm{D}(\mathrm{E}))$; in OFF channels, the excitation $(\mathrm{E})$ is computed via convolving surrounding delayed inhibitions $(\mathrm{D}(\mathrm{I})$ ); excitations and inhibitions compete with each other in local summation (S) cells; the grouping $(\mathrm{G})$ layer convolves excitations from $\mathrm{S}$ cells. (c) Spatiotemporal convolutions in PNNs.

\subsection{Photoreceptors}

In the LGMD1 model, as shown in Fig. 3a, the first layer consists of photoreceptors arranged as a 2D matrix. The total number of photoreceptors correspond to the number of local pixels $(n)$ in the field of view. Photoreceptors capture grey-scaled brightness and compute the change of luminance between 
Table 1: The LGMD1 neuron model components

\begin{tabular}{l|l|l|l|}
\hline \multicolumn{3}{c}{ acronym \& full-name } \\
\hline $\mathrm{P}$ & photoreceptor & $\mathrm{n}$ & total number of pixels \\
$\mathrm{LP}$ & low-pass filter & $\mathrm{PNN}$ & partial neural network \\
$W_{i}$ & inhibitory convolution matrix & $W_{e}$ & excitatory convolution matrix \\
$\mathrm{E} / \mathrm{I}$ & local excitation/Inhibition & $\mathrm{S} / \mathrm{G}$ & summation/grouping cells \\
$W_{g}$ & grouping matrix & $\mathrm{FFI}$ & feed forward inhibition \\
$\mathrm{SMP}$ & sigmoid membrane potential & $\mathrm{SFA}$ & spike frequency adaptation \\
\hline
\end{tabular}

every two successive frames:

$$
P(x, y, t)=L(x, y, t)-L(x, y, t-1)+\sum_{i}^{N_{p}} a_{i} \cdot P(x, y, t-i),
$$

where $P(x, y, t)$ is the brightness change corresponding to each local pixel: $x$ and $y$ are the abscissa and ordinate, $t$ indicates the current frame. $L(t)$ and $L(t-1)$ are the grey-scaled brightness of two successive frames. In addition, the luminance change could last for a short duration of $N_{p}$ number of frames. We defined a coefficient $a_{i}$ to be calculated by $a_{i}=\left(1+e^{u \cdot i}\right)^{-1}$ and $u=1$, simulating the fast (exponential) decay of residual luminance change.

\section{2. $O N$ and $O F F$ rectifying transient cells}

Next, the relayed visual signals from photoreceptors are split into parallel $\mathrm{ON}$ and $\mathrm{OFF}$ visual pathways via the mechanisms of $\mathrm{ON}$ and $\mathrm{OFF}$ rectifying transient cells (RTCs), encoding luminance increments (onset response) and decrements (offset response), respectively. Technically speaking, as shown in the PNN of LGMD1 model (Fig. 3b), the functionality of these polarity cells is reconciled with a 'half-wave' rectifier, which filters out negative/positive inputs for $\mathrm{ON}$ and OFF pathways, and inverts negative inputs to positive in the OFF pathway. Each photoreceptor corresponds to a pairwise ON and OFF RTCs:

$$
\begin{aligned}
& O N(x, y, t)=(P(x, y, t)+|P(x, y, t)|) / 2+\sigma_{p} \cdot O N(x, y, t-1), \\
& O F F(x, y, t)=|(P(x, y, t)-|P(x, y, t)|)| / 2+\sigma_{p} \cdot O F F(x, y, t-1) .
\end{aligned}
$$


We also allow a small fraction $\left(\sigma_{p}\right)$ of original polarity signals at previous frame in parallel to pass through to the following circuits mimicking the absolute brightness in the motion detection circuitry [41].

\subsection{Spatiotemporal visual processing in $O N$ and OFF pathways}

After 'half-wave' rectifying, the ON cells convey brightness increments to the ON pathway including the excitation $(E)$, the inhibition $(I)$ and the local ONsummation $\left(S_{o n}\right)$ cells, as shown in Fig. 3b. ON cells elicit onset responses, i.e., excitations are time-advance relatively to inhibitions and transmitted directly to the excitation cells in the ON pathway:

$$
E_{\text {on }}(x, y, t)=O N(x, y, t)
$$

Meanwhile, it is delayed by tens to hundreds of milliseconds, the mechanism of which is reconciled with a first-order low-pass filtering:

$$
\frac{d D_{o n}(x, y, t)}{d t}=\frac{1}{\tau_{s}}\left(O N(x, y, t)-D_{o n}(x, y, t)\right),
$$

where $\tau_{s}$ is a dynamic time parameter, which can vary between tens to hundreds of milliseconds in the low-pass filter. Inhibitions in the ON pathway are formed by convolving surrounding delayed excitations, as shown in Fig. 3c. Compared with previous LGMD1 models (e.g. [27, 15]), wherein the inhibition was computed by convolving surrounding one-frame-delayed excitations, we propose a dynamic spatiotemporal convolution: the nearest four neighboring cells share relatively higher weightings and shorter delays than the four diagonal cells. The temporal dynamics are illustrated in Fig. 3c, and the weightings of convolution kernel $\left(W_{i}\right)$ fits the following matrix:

$$
\left[W_{i}\right]=\left[\begin{array}{ccc}
1 / 8 & 1 / 4 & 1 / 8 \\
1 / 4 & 0 & 1 / 4 \\
1 / 8 & 1 / 4 & 1 / 8
\end{array}\right] .
$$

It is worth noticing that the delayed information only spreads out to their neighboring cells rather than to its direct counterpart. In this modeling study, 
the radius $(r)$ of convolution kernel is set to 1 , for the purpose of saving computational power, as the convolution process goes through each local cell in both ON and OFF pathways. Therefore, the inhibition in each interneuron of the ON pathway is calculated by the following equation:

$$
I_{o n}(x, y, t)=\sum_{i=-r}^{r} \sum_{j=-r}^{r} D_{o n}(x+i, y+j, t) \cdot W_{i}(i+r, j+r) .
$$

The OFF pathway processes visual information similarly to the ON pathway. However, since OFF cells elicit offset responses by brightness decrements, inhibitions are directly conveyed to the inhibition cells, whilst the excitation is formed by convolving surrounding delayed inhibitions, as illustrated in Fig. 3b and Fig. 3c. In this modeling study, we set the excitatory convolution kernel ([$\left.\left.W_{e}\right]\right)$ equal to the $\left[W_{i}\right]$ in Eq. 5. The dynamic temporal parameter $\tau_{s}$ is used to filter inhibitions in the OFF pathway as well:

$$
\frac{d D_{o f f}(x, y, t)}{d t}=\frac{1}{\tau_{s}}\left(\operatorname{OFF}(x, y, t)-D_{o f f}(x, y, t)\right) .
$$

Accordingly, the excitation and inhibition are calculated as follows:

$$
\begin{aligned}
& I_{o f f}(x, y, t)=\operatorname{OFF}(x, y, t), \\
& E_{o f f}(x, y, t)=\sum_{i=-r}^{r} \sum_{j=-r}^{r} D_{o f f}(x+i, y+j, t) \cdot W_{e}(i+r, j+r) .
\end{aligned}
$$

After that, each polarity summation cell linearly integrates excitation and inhibition with the biases $w_{1}$ and $w_{2}$, in order to suppress each inhibitory flow:

$$
\begin{aligned}
& S_{o n}(x, y, t)=E_{o n}(x, y, t)-w_{1} \cdot I_{o n}(x, y, t), \\
& S_{o f f}(x, y, t)=E_{o f f}(x, y, t)-w_{2} \cdot I_{o f f}(x, y, t) .
\end{aligned}
$$

\subsection{Summation and grouping layers}

With similar ideas in a few biological and computational modelling studies on a fly's visual system, 42, 45, 43, the relayed local excitations from ON and OFF channels interact with each other in a supralinear (both multiplicative and linear) way at each summation cell in the PNN ( $S$ in Fig. 3b):

$$
S(x, y, t)=\theta_{1} \cdot S_{o n}(x, y, t)+\theta_{2} \cdot S_{o f f}(x, y, t)+\theta_{3} \cdot S_{o n}(x, y, t) \cdot S_{o f f}(x, y, t),
$$


where $\left\{\theta_{1}, \theta_{2}, \theta_{3}\right\}$ indicates the combination of term coefficients, which allows us to represent different 'balances' of interactions between ON and OFF pathways and realise either purely linear or nonlinear computation. Importantly, such a formula can summarise two computational principles in the modelling of biological motion sensitive systems. For example, the multiplicative computations have been applied in models based on Hassenstein-Reichardt Correlation (HRC) detectors, like the elementary motion detectors in flies (e.g. 62, 7, 31, 50]). The purely linear computations have been used in previous locust LGMD1 and DSNs neuronal models, e.g. [27, 63, 64, 65, 15. More specifically, in the LGMDs neuron models, such a supralinear computation manner that has also been used to separate the different looming selectivity between LGMD1 and LGMD2 21].

In this LGMD1 neuron model, we implement the selectivity to expanded edges by clustering excitations of looming objects, through a simplified grouping layer ( $G$ in Fig. 3b) relative to the similar LGMD1 models 27, 15] in Fig. 2a It is basically a convolution course:

$$
G(x, y, t)=\sum_{i=-r}^{r} \sum_{j=-r}^{r} S(x+i, y+j, t) \cdot W_{g}(i+r, j+r),
$$

where $W_{g}$ is an equal-weighted kernel as shown in Fig. 3c, and the radius of convolving area is also set to 1 :

$$
\left[W_{g}\right]=\frac{1}{9}\left[\begin{array}{lll}
1 & 1 & 1 \\
1 & 1 & 1 \\
1 & 1 & 1
\end{array}\right] .
$$

For the grouped cells, the clustered and stronger excitations will pass through to the LGMD1 cell, whilst the smaller isolated (or decayed) excitations are eliminated by thresholding:

$$
G^{\prime}(x, y, t)=\left\{\begin{array}{cl}
G(x, y, t), & \text { if } G(x, y, t) \geq T_{g} \\
0, & \text { else }
\end{array}\right.
$$

255 3.5. LGMD1 cell

At the LGMD1 cell, the neural processing is a competition between the feedforward excitation and the feed-forward inhibition: if the excitation wins, the 
neuron is activated to generate spikes, otherwise, it is rigorously inhibited. The feed-forward excitation is formed by linearly pooling all local excitations from the grouping layer which can be represented by the membrane potential in the terminology of biology:

$$
M P(t)=\sum_{x=1}^{\text {row }} \sum_{y=1}^{\text {col }} G^{\prime}(x, y, t),
$$

where row and col are the numbers of rows and columns of the grouping layer. The membrane potential is then exponentially mapped to sigmoid membrane potential, the transformation of which mimics the activation of artificial neurons:

$$
U(t)=\left(1+e^{-|M P(t)|\left(n \cdot K_{s i g}\right)^{-1}}\right)^{-1},
$$

where $U$ indicates the sigmoid membrane potential (SMP in Fig. 3) and $n$ denotes the total number of photoreceptors. The output is thus normalised within $[0.5,1)$ with a scale parameter of $K_{\text {sig }}$ towards the following spike mechanism.

On the other hand, the feed-forward inhibition is formed in a parallel pathway relatively to the whole pre-synaptic area (Fig. 1 and Fig. 3a. Like the former LGMD1 models [10, 27, 57, 15], the FFI mechanism obeys an 'all-ornone' law, meaning it can directly suppress the LGMD1 cell if a large area of luminance change occurs rapidly within the field of view. The mathematic expression of FFI is taking the average value of absolute luminance change captured by photoreceptors:

$$
F(t)=\sum_{x=1}^{\text {row }} \sum_{y=1}^{\text {col }}|P(x, y, t)| \cdot n^{-1} .
$$

It is then delayed by tens to hundreds of milliseconds with a time parameter $\tau_{f}$ in the low-pass filtering:

$$
\frac{d F^{\prime}(t)}{d t}=\frac{1}{\tau_{f}}\left(F(t)-F^{\prime}(t)\right)
$$

Once the postponed FFI output exceeds a predefined threshold level $\left(T_{f f i}\right)$, the generated membrane potential is cut off directly and LGMD1 neuron is inhibited 
immediately, otherwise, the FFI has no effects on the LGMD1 cell:

$$
U(t)=0.5, \text { if } F^{\prime}(t) \geq T_{f f i}
$$

where the sigmoid membrane potential is set to its minimum value of, in our case, 0.5 .

\subsection{Spike frequency adaptation}

As presented in above sections, in order to further enhance the visual looming selectivity, we computationally model the biophysical SFA mechanism. Its computational role allows a neural response with a positive derivative profile to overcome adaptation selectively, otherwise, the neural response is heavily blocked causing a quick decline. Such a mechanism can be mathematically defined as:

$$
U^{\prime}(t)=\left\{\begin{array}{l}
\sigma_{\text {slow }} \cdot U(t), \text { if } d^{2} U(t) / d t^{2} \geq 0 \\
\sigma_{\text {fast }} \cdot U(t), \text { if } d^{2} U(t) / d t^{2}<0 \& d U(t) / d t \geq 0 \\
\sigma_{\text {fast }} \cdot\left(U^{\prime}(t-1)+U(t)-U(t-1)\right), \text { if } d U(t) / d t<0
\end{array}\right.
$$

where $\sigma_{\text {slow }}$ and $\sigma_{\text {fast }}$ denote two coefficients simulating 'slow' and 'fast' adaptations respectively, which can be calculated as:

$$
\sigma_{\text {slow }}=\tau_{\text {slow }} /\left(\tau_{\text {slow }}+\tau_{i}\right), \quad \sigma_{\text {fast }}=\tau_{\text {fast }} /\left(\tau_{\text {fast }}+\tau_{i}\right) .
$$

$\tau_{\text {slow }}, \tau_{\text {fast }}$ indicate two time constants in milliseconds. $\tau_{\text {slow }}$ is greater than $\tau_{\text {fast }}$ and $\tau_{i}$ is the time interval, also in milliseconds, between successive frames. It is worth emphasising that as the digital signals do not have continuous derivatives, we compute the gradient by comparing signals at successively discrete frames. It is also necessary to notice that the delays $\left(\tau_{\text {slow }}\right.$ and $\left.\tau_{\text {fast }}\right)$ could vary within a wide range from hundreds to thousands of milliseconds in order to partition adaptation rates for different profiles of the LGMD1 neural response. 


\subsection{Spiking mechanism}

After the SFA mechanism, a different number of spikes could be generated at each time point by an exponential mapping from the neural membrane potential to the firing rate:

$$
S^{s p i k e}(t)=\left\lfloor e^{\left[K_{s p} \cdot\left(U^{\prime}(t)-T_{s p}\right)\right]}\right\rfloor,
$$

where $\lfloor x\rfloor$ indicates a function to return the largest integer less than or equal to the input $x . K_{s p}$ and $T_{s p}$ denote a scale parameter and a threshold in the spiking mechanism: increasing $K_{s p}$ will lead to higher spike frequency with an identical input. As a result, compared with previous works on LGMD1 modelling, such as [27, 15, there could be more than one spike at each frame being generated. Finally, a potential collision recognition is given by:

$$
\operatorname{Col}(t)=\left\{\begin{array}{l}
\text { true, if } \sum_{i=t-N_{t}}^{t} S^{s p i k e}(i) \geq N_{s p} \\
\text { false, otherwise }
\end{array},\right.
$$

where $N_{s p}, N_{t}$ denote the number of successive spikes and frames, respectively. We set $N_{s p}$ to be greater than $N_{t}$ in this modelling study, as the exponential mapping from membrane potential to firing rate. As depicted in Fig. 1 and $3 \mathrm{a}$ the generated spikes by the LGMD1 neuron are conveyed to its post-synaptic target-neuron, the DCMD, and towards further motion neural systems for collision avoidance behaviours.

\subsection{Parameters setting}

All of the parameters of the proposed visual neural network are decided empirically with considerations and optimisations of the underlined functionality of $\mathrm{ON}$ and $\mathrm{OFF}$ pathways and the SFA mechanism to implement the underlying characteristics of a biological LGMD1 as an embedded vision system for a ground miniaturised robot. Table 2 illustrates the parameters' settings: all the adaptable parameters correspond to the physical properties of the input visual streams, i.e., the resolution of images and the sampling frequency of 
Table 2: The predefined parameters of LGMD1 neuron model

\begin{tabular}{|ll|ll|ll|}
\hline Name & Value & Name & Value & Name & Value \\
\hline col, row & adaptable & $K_{s p}$ & 4 & $\tau_{s}$ & $15 \sim 120(\mathrm{~ms})$ \\
$w_{1}$ & 0.3 & $w_{2}$ & 0.6 & $\tau_{i}$ & adaptable \\
$n$ & col $\cdot$ row & $K_{\text {sig }}$ & 1 & $\tau_{f a s t}$ & $300 \sim 500(\mathrm{~ms})$ \\
$\theta_{1}$ & $1 \sim 2$ & $\theta_{2}$ & $0.5 \sim 1$ & $\theta_{3}$ & $0 \sim 0.6$ \\
$T_{s p}$ & $0.66 \sim 0.74$ & $N_{t}$ & 4 & $N_{s p}$ & $4 \sim 8$ \\
$\tau_{f}$ & $10 \sim 100(\mathrm{~ms})$ & $T_{g}$ & 10 & $T_{f f i}$ & 10 \\
$\sigma_{p}$ & 0.1 & $\tau_{\text {slow }}$ & $700 \sim 1000(\mathrm{~ms})$ & $r$ & 1 \\
\hline
\end{tabular}

micro-robot. It is worth emphasising that the parameters learning and training methods are not applied to this neuron model. Compared to previous LGMD1 studies [15, 27], we will investigate and demonstrate the effects of several neuron model parameters on collision detection, including the spiking threshold as well as temporal parameters for the ON and OFF pathways and SFA mechanism in the next section of experiments.

\section{Experiments and results}

In this section, we will present our experiments. All of the experiments can be categorised into two types, off-line and on-line. For off-line, we tested the proposed framework using synthetic stimuli and physical stimuli. For comparison, we compared its performance with a previous LGMD1 computational model [27, 15]. We also compared the proposed model with the biological data and a biological LGMD1 model [46, 47]. For the on-line tests, the proposed framework was embedded into a visual module of a ground micro-robot for both arena tests and other investigations. The main objectives are as follows: firstly, to examine the effectiveness and robustness of the proposed LGMD1 neuron model in collision detection and, secondly, to provide insights into the underlined mechanisms of ON and OFF pathways and SFA in shaping the collision selectivity. 


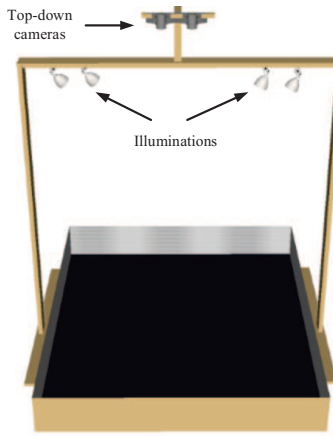

(a) Arena

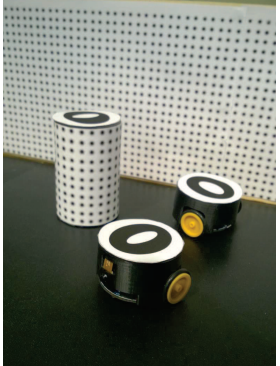

(b) Robot and obstacle

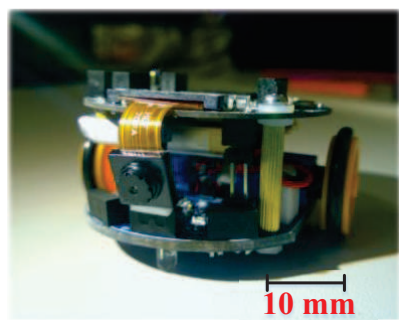

(c) Robot prototype

Figure 4: Illustrations of the arena and Colias the robot used in the on-line tests: (a) The arena profile, (b) A subregion view of the arena with Colias robots and obstacles, (c) The Colias robot prototype.

\subsection{Experimental set-up}

Software set-up. The off-line tests, proposed framework and comparative LGMD1 model [27, 15] were all set up in Visual Studio 2015 (Microsoft Corporation). Data analysis and visualisations were generated in Matlab 2015b (The MathWorks, Inc. Natick, USA). The resolution of input synthetic stimuli of loomingreceding, translating and sinusoidal-grating movements are $300 \times 300,400 \times 200$ 30 and $320 \times 240$, respectively, and were all at $30 \mathrm{fps}$. The resolution of real-world stimuli is $352 \times 288$ at $23 \mathrm{fps}$. Model parameters of the proposed framework corresponded to Table 2, whilst the parameters of the comparative LGMD1 model were obtained from previous research [27, 15].

Hardware set-up. In the on-line tests, a low-cost monocular vision based microrobot named 'Colias' 66, 15, with an RGB-camera sensing module, was used. This is the only applied sensor in this research. It has been developed for biorobotics research, including swarm robotic applications [67, 66], as well as neural vision systems research [56, 15, 21, 20, 9]. As illustrated in Fig. 4c, the robot has a small footprint measuring only $4 \mathrm{~cm}$ in diameter and $3 \mathrm{~cm}$ in height, with two main modules. The bottom board is the motion actuator with two differential DC motors that provide the platform with a maximum speed of approximately 
$35 \mathrm{~cm} / \mathrm{s}$. In addition, a $3.7 \mathrm{~V}, 320 \mathrm{mAh}$ lithium battery supports the autonomy for $1 \sim 2$ hours.

The upper board executes vision-based models. Its processor used to run detections.

\subsection{Off-line tests}

Challenged by synthetic stimuli. First of all, the experiments started by testing the proposed LGMD1 model using synthetic stimuli and comparing its looming uli can be categorised into the following types: approaching-receding (Fig. 6), translating (Fig. 7) and sinusoidal gratings (Fig. 10). There is no environmental noise in the synthetic scenes. We also compared the results with the neural response of biological LGMD1 neuron and model [46, 47] (Fig. 5), by using

challenged by grating movements with a broad range of spatial and temporal 


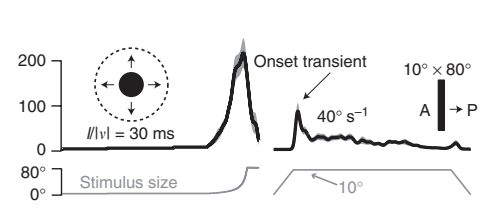

(a)

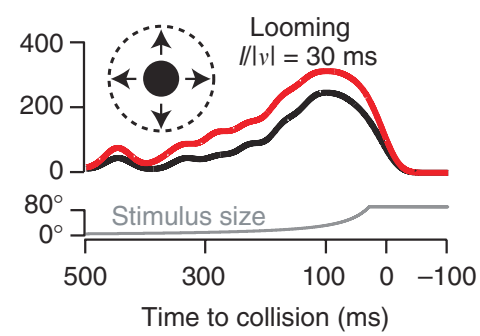

(d)

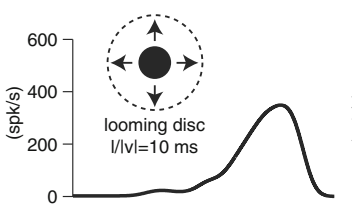

(b)

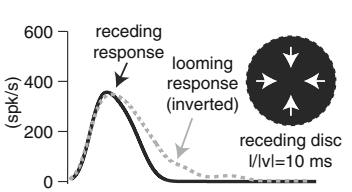

(c)

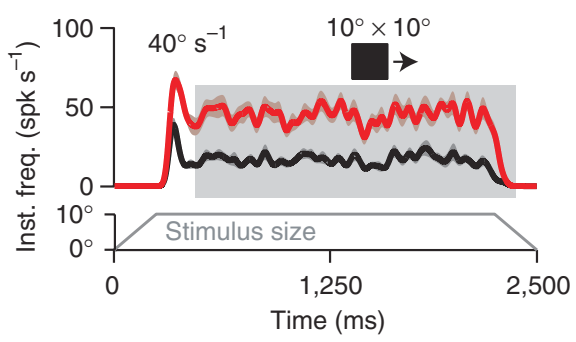

(e)

Figure 5: Neural response of a biological LGMD1 neuron or model adapted from [46 47]: (a) Biological LGMD1 neuronal response to looming and translation. (b) Biological LGMD1 model response to looming. (c) Biological LGMD1 model response to recession. The LGMD1 model shows asymmetric responses that the response is quickly decayed by receding stimuli. (d) Biological model response to looming stimuli without (red-curve) and with (black-curve) the SFA mechanism. (e) Biological model response to translation stimuli. The LGMD1 neuron overcomes adaptation by looming with an increasing intensity of stimuli, yet the response is largely weakened by translation with a constant intensity.

frequencies, which were reconciled with visual clutter in the real world.

In the first part of synthetic tests, we examined if the proposed LGMD1 model possesses similar characteristics to a biological LGMD1 neuron. Fig. 3555 illustrates the biological LGMD1 neuron and model response by looming, receding, and translating stimuli, which reveals three important points: (1) the LGMD1 neuron can overcome adaptation in looming; (2) the LGMD1 represents asymmetric response at the end of looming and the start of recession, i.e., the neural response decays quickly by receding; (3) the response of LGMD1 decays quickly by translation at a constant speed. Our results in Fig. 6 and Fig. 7 show that the proposed LGMD1 neuron model has demonstrated all of these characteristics. On the other hand, when challenged by looming and receding stimuli, the comparative LGMD1 model demonstrates symmetrical responses. 

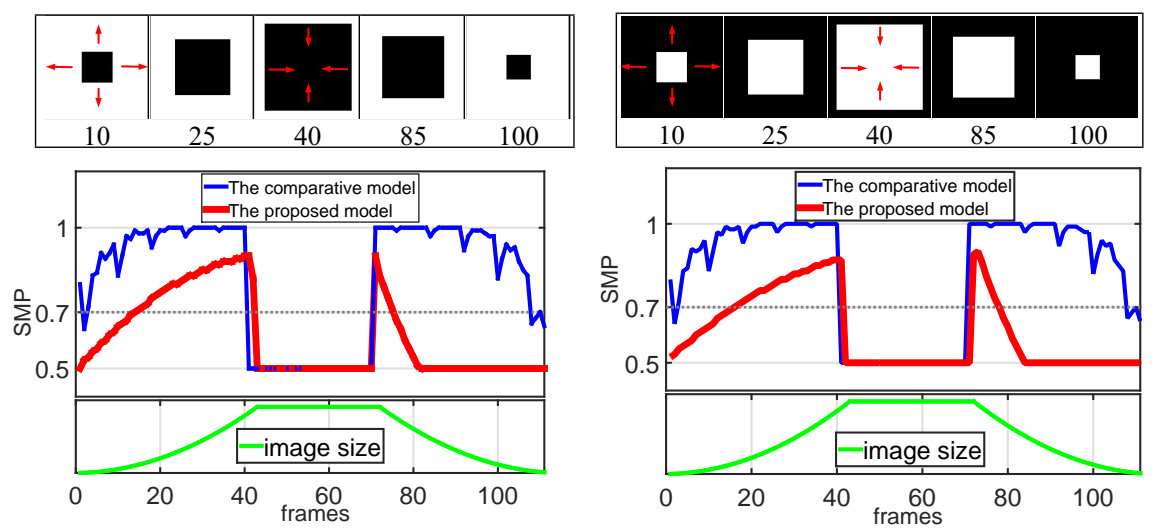

Figure 6: Neural responses of the proposed LGMD1 model and the comparative model by synthetic looming and receding movements of a dark and a light objects embedded on light and dark backgrounds, respectively. The image size is depicted at the bottom. The snapshots are shown at top. Y-axis indicates the SMP. X-axis denotes the time window in frames. The horizontal dashed-lines designate the spiking threshold. Both models show continuous increasing response by the looming stimuli; while the response of the proposed model decays very quickly in recession.
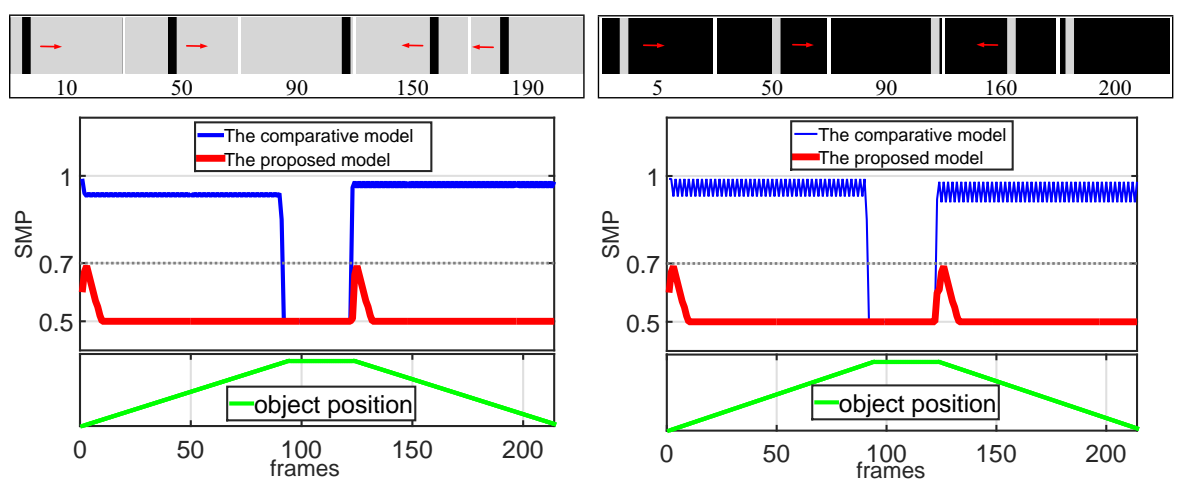

Figure 7: Neural response of the proposed LGMD1 model and the comparative model by synthetic dark/light translating movements. The object-position is indicated at the bottom of the result. The proposed LGMD1 model demonstrates a much weaker and quickly decayed response to translations at constant speeds in both directions; while the comparative LGMD1 model shows continuously stronger response to translations. 

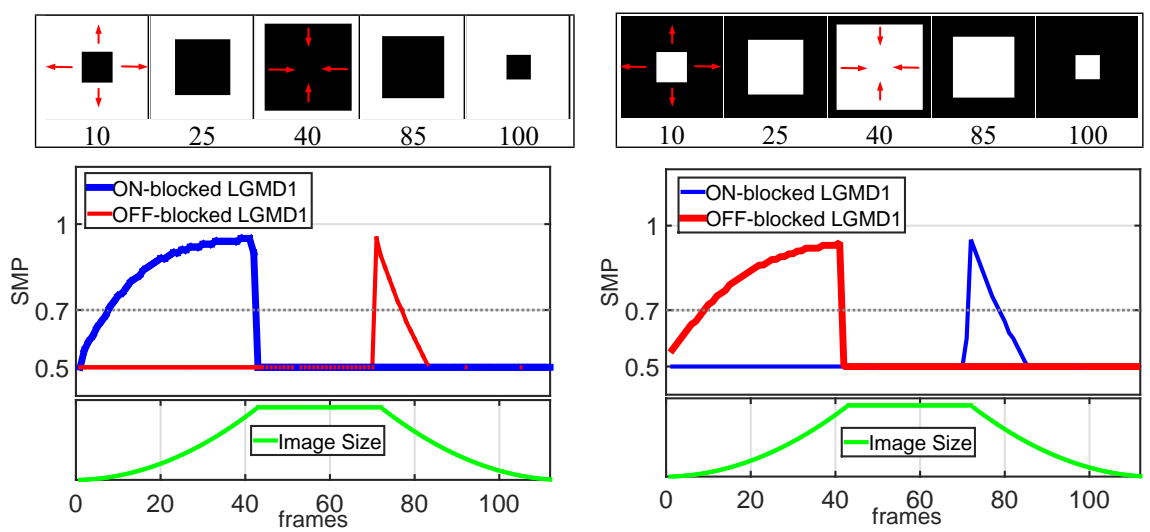

Figure 8: Neural response of the proposed LGMD1 model challenged by the similar looming and receding stimuli to Fig. 6 6 yet with $\mathrm{ON}$ and OFF pathways alternately being blocked.

Blocking the ON pathway abolishes the onset response by luminance increments and can realise the underlying functionality of a biological LGMD2; while blocking the OFF pathway abolishes the offset response by luminance decrements.

It also appears that the comparative LGMD1 model is affected by translations more significantly with continuously high-level neural responses. Therefore, compared with the previous LGMD1 neuron model [27, the collision selectivity is effectively enhanced for looming rather than receding and translating stimuli. The neural response of the proposed model is consistent and match the results in Fig. 5

In the second part of synthetic tests, we investigated the functionality of the ON and OFF visual pathways in the proposed LGMD1 model. As illustrated in Fig. 8, we blocked either the ON or OFF pathways in looming and receding tests. Interestingly, the results demonstrate that blocking the ON pathway rigorously abolishes the underlying functionality of $\mathrm{ON}$ polarity cells for the onset response 375 by luminance increments, i.e., the LGMD1 model only responds to the dark object looming and the light object receding. While after blocking the OFF pathway, the model is sensitive to only dark object receding and light object looming. Our previous research (Fig. 2b) has demonstrated that such a bioplausible structure has great potential to realise the underlying functionality of a 


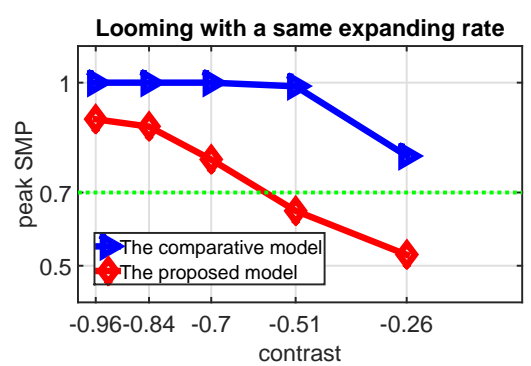

(a)

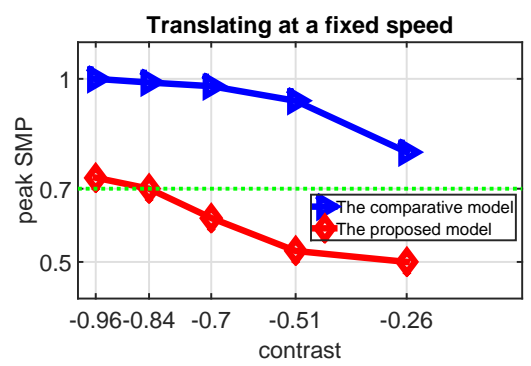

(c)

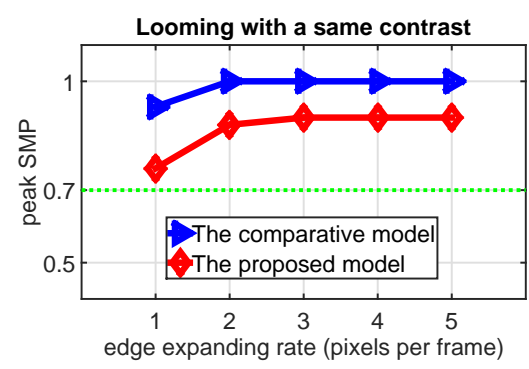

(b)

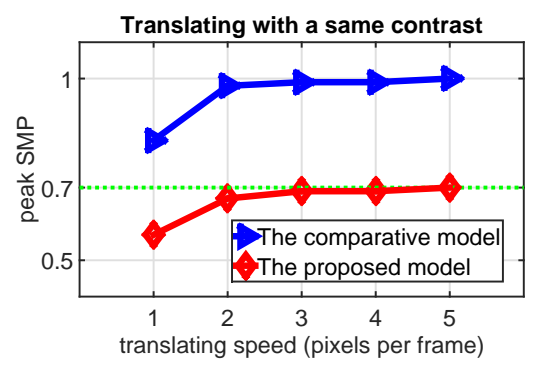

(d)

Figure 9: Peak neural responses of the proposed (red) and comparative (blue) LGMD1 models by synthetic looming and translation movements of dark objects in a bright background. The stimuli of dark objects are with different contrasts to the background and moving at different speeds. The green dashed lines indicate the spiking threshold. The proposed LGMD1 model demonstrates contrast sensitivity and speed response to looming and translating stimuli, better than the comparative LGMD1 model. The proposed model is not significantly activated by translations at constant speeds.

biological LGMD2 neuron, which is only sensitive to the light-to-dark luminance change, and consistent with the proposed ON-blocked LGMD1 model in Fig. 8

Furthermore, we systematically investigated the effects of two basic properties of visual stimuli on neuronal responses of the proposed LGMD1, the speed and the contrast, and comparative model for the movement of dark objects approaching and translating. In this case, we define the contrast between the moving objects and the background to be calculated by:

$$
\text { Contrast }=\left(L_{o b j}-L_{b a c k}\right) / L_{b a c k},
$$

where $L_{o b j}$ and $L_{b a c k}$ are the average luminance of the moving object and the 
background. The results in Fig. 9 allows the following conclusions to be drawn: both LGMD1 models represent comparable speed response. The neural responses all steadily peak at higher levels when the approach and translation movements speed up (Fig. 9b and 9d). By fixing the edge expanding rate of looming objects (Fig. 9a), the peak responses of both models reach a valley with the smallest contrast. And compared with the former LGMD1 model, the proposed model demonstrates more significant reduction of peak response. The proposed LGMD1 model could fail to perceive looming stimuli once the contrast decreases below $|-0.51|$ (Fig. 9a), which validates that the proposed LGMD1 model is more sensitive to the contrast between moving objects and the background.

Challenged by translations, the results in Fig. 9c, by movements at fixed speeds, reveal the proposed framework could also treat the translations, with larger contrasts or at faster speeds, as potential collisions. Intuitively, when challenged against translation movements, the proposed LGMD1 model is not easily activated like the previous LGMD1 model, as the neural response is largely weakened. To briefly summarise, compared with the comparative LGMD1 model, the proposed bio-plausible mechanisms and spatiotemporal computations play roles in shaping the LGMD1's collision selectivity to looming rather than translation.

In the last part of the synthetic tests, we examined the performance of the proposed neuron model against gratings with a wide range of spatial and tempo405 ral frequencies. In previous biological research 10, 11, 13, these locust looming detectors have proposed robust performance against gratings corresponding to visual clutter in the real world. The locust LGMD1 neuron is rigorously inhibited by gratings. Fig. 10 illustrates that we have achieved similar results to previous biological findings, which is a critically important ability for a practical collision detector. In the proposed neuron model, we realise such an ability by low-level spatiotemporal visual processing, instead of the registration or classification based methodologies. 


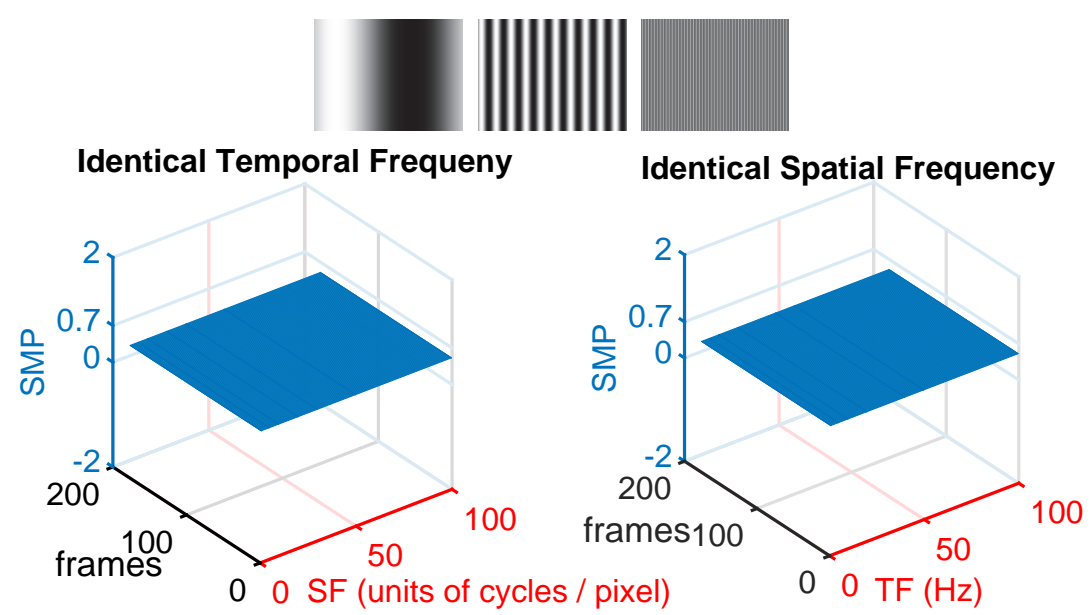

Figure 10: Neural responses of the proposed LGMD1 model are challenged by sinusoidal grating stimuli with a wide range of spatial and temporal frequencies (SF/TF), respectively. The example grating patterns, as input, are shown at the top. The spiking threshold is set at 0.7. The proposed LGMD1 model remains quiet by all tested gratings, similarly to a biological LGMD1 neuron in locusts.

Challenged by real physical stimuli. In the second set of off-line tests, we also gave an initial insight into the efficacy of proposed collision perception vision systems in ground vehicle applications. We used on-road recordings from dashboard cameras as the visual stimuli to test the LGMD1 model. As illustrated in Fig. 11 and Fig. 12, the input from off-line stimuli involved both colliding and non-colliding driving scenarios in complex and dynamic scenes, which are frequent visual challenges to drivers.

In the first case, as shown in Fig. 11, our results demonstrate that the proposed LGMD1 model successfully recognises these impending vehicle-collisions: the LGMD1 neuron is highly activated by these fast approaching stimuli. Interestingly, the FFI can also indicate a potential collision, which increases dramatically before colliding. A defect of the current model is that the predefined FFI threshold influences the collision-detecting ability since the FFI can directly suppress the LGMD1 neuron in the critical moments before the end of the rapidly approaching stimuli in this neuron model. Therefore, an automated 


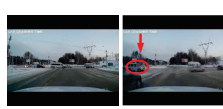

10

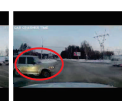

150

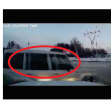

160

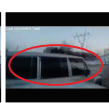

170

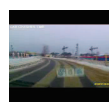

20

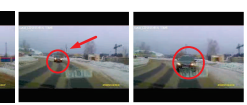

110

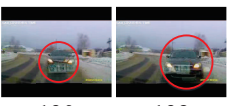

130

132
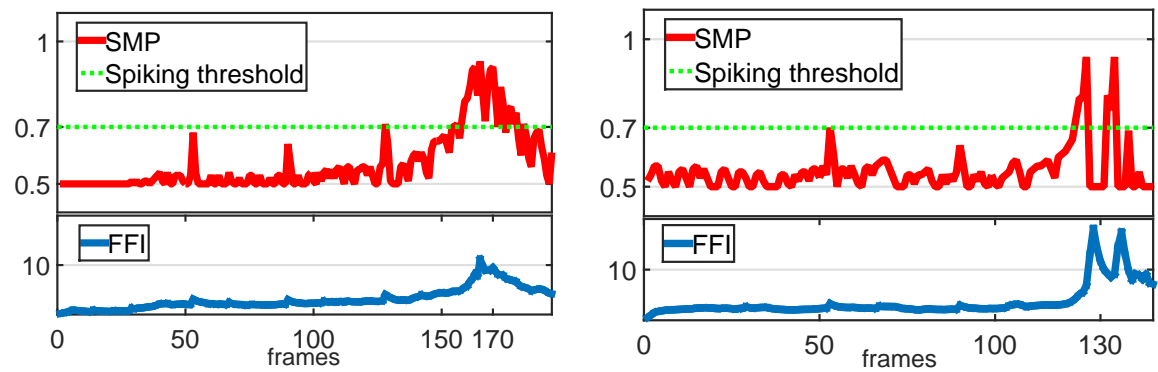

Figure 11: Neural responses of the proposed LGMD1 model challenged by 'colliding scenarios' of real-world stimuli from recordings of ground-vehicle dashboard cameras. The snapshots are shown at each top. The proposed LGMD1 neuron model successfully recognises the on-road impending collisions in complex and dynamic environments.

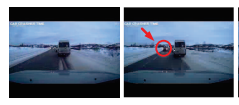

20

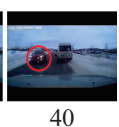

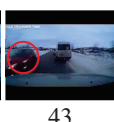

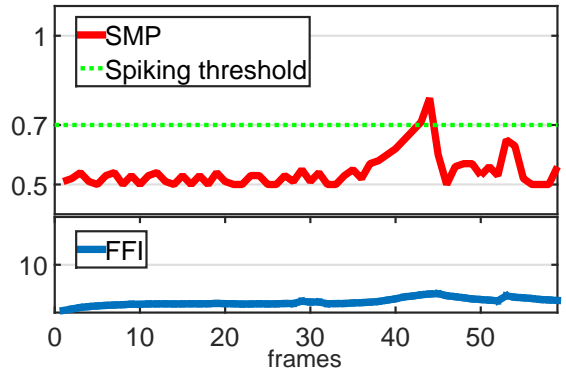

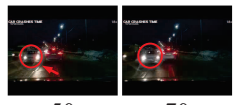

70

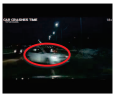

90

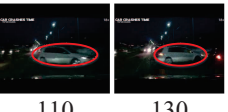

110

130

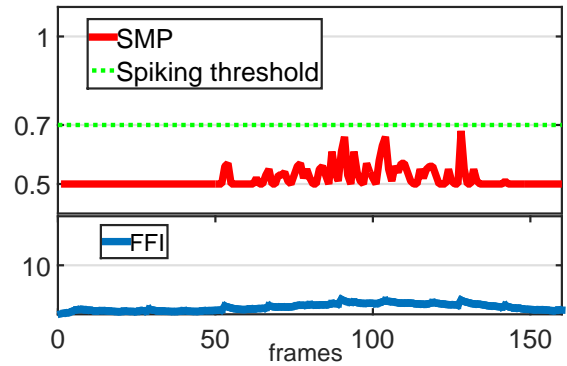

Figure 12: A neural response of the proposed LGMD1 model challenged by 'non-colliding scenarios' of real-world stimuli including 'near-miss' and translation scenes. The proposed LGMD1 model is not activated by the translation scene, yet only shortly activated by the 'near-miss' scene, both of which were correctly recognised as 'non-collision'.

adjusting of the FFI threshold is badly needed in the future research.

In the second case, to make the comparison, we also tested the proposed LGMD1 model with two non-colliding scenarios - a near-miss and a translation scene. Our results in Fig. 12 demonstrate that the proposed LGMD1 


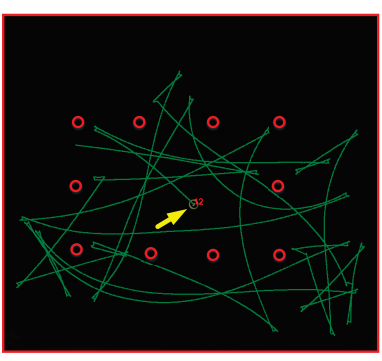

Linear speed: $5 \mathrm{~cm} / \mathrm{s}$

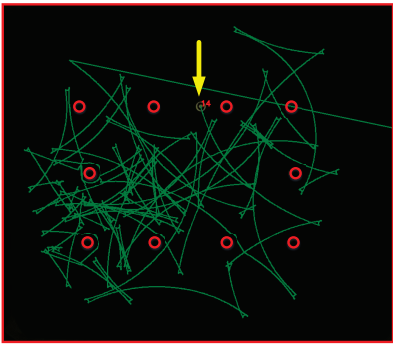

Linear speed: $10 \mathrm{~cm} / \mathrm{s}$

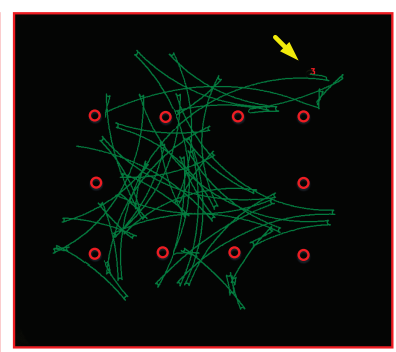

Linear speed: $15 \mathrm{~cm} / \mathrm{s}$

Figure 13: Example results of arena tests with overtime trajectories of a Colias robot implementing the proposed LGMD1 neuron model. The yellow arrow indicates the end-position of the robot with the specific ID in each arena test; the obstacles are indicated by red circles for the same layout. The Colias robot was tested at different linear speeds. The results demonstrate its efficacy for collision detection in robot navigation applications.

neuron model successfully recognises these movements as non-collision events. Our initial tests with driving scenarios can provide important implications for the future research on applying the locust looming sensitive vision systems to build neuromorphic sensors in the applications of ground vehicles for improving driving safety.

\subsection{Robot tests}

In this subsection, we continue to present our on-line bio-robotics experiments. The proposed LGMD1 neuron model was implemented in the monocular vision based Colias robot. We applied the camera sensor as the only utilised modality for collision detection. To examine its performance in robotic applications and deepen the understandings of the underlined bio-plausible mechanisms in shaping LGMD1's collision selectivity, we designed two kinds of on-line tests: the arena tests and the other systematic tests.

\subsubsection{Arena tests}

In the first type of on-line tests, we inspected the basic collision-detecting ability of the proposed method in an arena with many obstacles. In the arena tests, we investigated the effects of linear-speed of the Colias robot on the 
Table 3: Success rates of collision avoidance

Success Rate(SR), Correct Avoidance(CA), Miss Avoidance(MA)

\begin{tabular}{|l|l|l|l|l|l|}
\multicolumn{7}{c}{$S R=C A /(C A+M A) \cdot 100 \%$} \\
\hline linear speed(cm/s) & 3 & 5 & 10 & 15 & 20 \\
\hline CA & 61 & 115 & 306 & 322 & 460 \\
\hline MA & 18 & 19 & 34 & 14 & 20 \\
\hline SR & $77.2 \%$ & $85.8 \%$ & $90.0 \%$ & $95.8 \%$ & $95.8 \%$ \\
\hline
\end{tabular}

Table 4: Comparative success rates of collision avoidance

\begin{tabular}{|l|l|l|l|l|l|}
\hline LGMD1 neuron models & $3(\mathrm{~cm} / \mathrm{s})$ & 5 & 10 & 15 & 20 \\
\hline \hline Proposed LGMD1 & $77.2 \%$ & $\mathbf{8 5 . 8 \%}$ & $\mathbf{9 0 . 0 \%}$ & $\mathbf{9 5 . 8 \%}$ & $\mathbf{9 5 . 8 \%}$ \\
\hline Comparative LGMD1 & $\mathbf{8 1 . 3 \%}$ & $83.6 \%$ & $85.0 \%$ & $88.8 \%$ & $87.4 \%$ \\
\hline Proposed (ON-blocked) & $79.0 \%$ & $85.0 \%$ & $86.5 \%$ & $92.7 \%$ & $89.0 \%$ \\
\hline Proposed (OFF-blocked) & $60.0 \%$ & $70.5 \%$ & $73.9 \%$ & $78.0 \%$ & $73.2 \%$ \\
\hline
\end{tabular}

success rate of collision detection and avoidance. The linear speeds thus varied from the slowest speed of $3 \mathrm{~cm} / \mathrm{s}$ to the fastest speed of $20 \mathrm{~cm} / \mathrm{s}$ as shown in Table 3. Moreover, for comparison, we also did arena tests for the comparative model [15], as well as the proposed neuron model with ON or OFF pathway blocked, respectively. The arena experimental setting was identical for each kind of LGMD1 neuron model. More specifically, in the arena tests, the Colias robot with each tested neuron model was initialised to go forward until a potential collision was detected. The collision-avoidance behaviour was simply set to turn right or left randomly with equal probability. After each avoidance, it resumed going forward. For all tested neuron models, the time window was set to 7 minutes for each test, whilst each speed test was repeated four times.

Firstly, Fig. 13 illustrates the trajectories of collision avoidance for the Colias robot with the proposed LGMD1 neuron model from our repeated tests. Moreover, Table 3 shows the statistical success rates of collision avoidance. We defined the 'miss avoidance' as any human interventions in the arena tests after 
the Colias robot got stuck at the edges of the arena or collided with an obstacle. The statistical results in Table 3 demonstrate that the Colias robot at the slowest linear speed $(3 \mathrm{~cm} / \mathrm{s})$ has the lowest success rate of collision avoidance in the arena tests. Increasing the linear speed gives rise to a rising of the success rate, which seems to peak around $15 \mathrm{~cm} / \mathrm{s}$ for the tested Colias robot. As a result, faster moving speeds make the visual neuron more sensitive to the looming stimuli by approaching the obstacles. However, we observed the larger distances to collisions (DTC) of the Colias robot at faster linear speeds.

For comparison, under the same arena test settings, we investigated the performance of the collision avoidance with the comparative LGMD1 neuron model, as well as the effects of blocking either ON or OFF pathways for the 475 proposed model with the collision avoidance. Table 4 demonstrates that our proposed LGMD1 model outperforms the comparative model in the arena tests, despite it being at the slowest speed of $3 \mathrm{~cm} / \mathrm{s}$. Interestingly, after blocking the ON pathway, the performance of the collision avoidance for the proposed model is only slightly affected; while the collision avoidance performance deteriorates sharply after blocking the OFF pathway in arena tests. The results demonstrate that the ON and OFF pathways play a role in the proposed neuron model for collision detection. Importantly, for the ground robotic navigation tests, the OFF pathway has a more significant influence on looming perception than the ON pathway, as most objects are darker than the background in the arena.

\subsubsection{Open-loop tests}

For systematically studying the unique characteristics of the proposed neuron model in robotic applications, we designed a few types of open-loop tests. The first kind of open-loop test was to test the proposed embedded LGMD1 model with movements in depth (Fig. 14). The second kind of open-loop test was to investigate the DTC response (Fig. 15 and 16). After that, we also studied the effects of angular approach (Fig. 18) and translation (Fig. 19) stimuli on the neural response of the proposed model. The experimental settings for the angular approach and translation tests are illustrated in Fig. 17. In the 

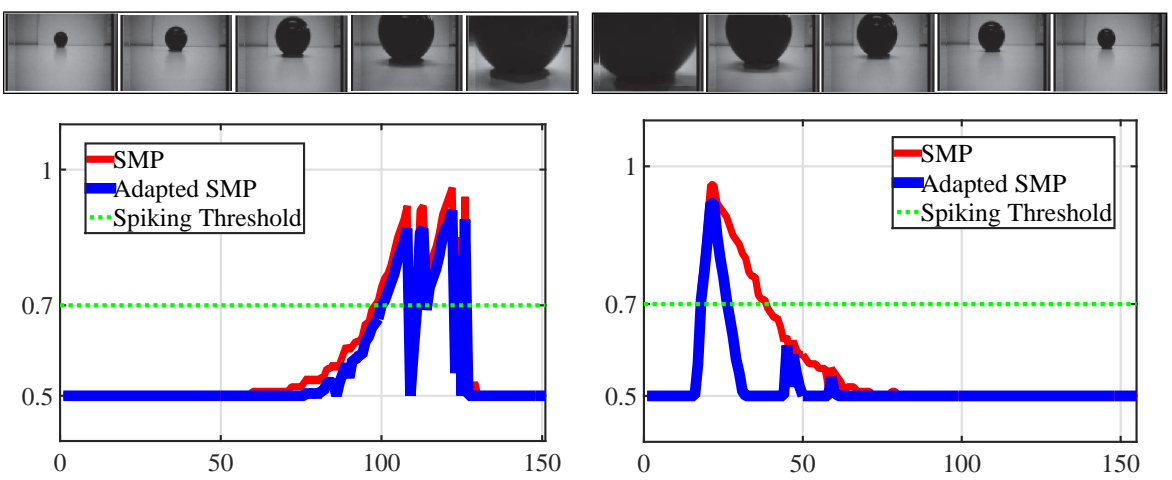

Figure 14: Neural responses of the embedded LGMD1 model in the stimulated Colias robot challenged by the looming and recession of a dark object. The first example views are shown at the top of each result. $\mathrm{X}$ and $\mathrm{Y}$ axes indicate the time window in frames and SMP from the tested robot. Both the neural responses before and after the proposed SFA mechanism are shown. The robot neural response matches the results in Fig. 5

two former test types, the motion controls unit was used for the Colias robot for approaching or receding from the targets; while in the angular approach and translation tests, the Colias robot was a motionless observer stimulated by another moving robot. In all the open-loop tests, we collected the neural response of the proposed model including the SMP and spikes through a Bluetooth device attached to the visual module of the tested Colias robot.

Firstly, Fig. 14 demonstrates that when challenged by direct looming stimuli caused by ego-motion of the Colias robot, the proposed LGMD1 model overcomes adaptation representing continuously increasing neural response as the size of the projected object grows in the field of view. It is fully activated by the end of approaching. The neural response is consistent and matches Fig. $5 \mathrm{a}$, $5 \mathrm{~b}$ $5 \mathrm{~d}$. On the other hand, when receding from the object, the proposed embedded LGMD1 model is only activated for a short period of time. The adapted neural response decreases dramatically, which matches Fig. 5c.

DTC tests. As mentioned in the arena tests, for deepening the understanding of the underlined correlations between the DTC and the looming speed, we also 


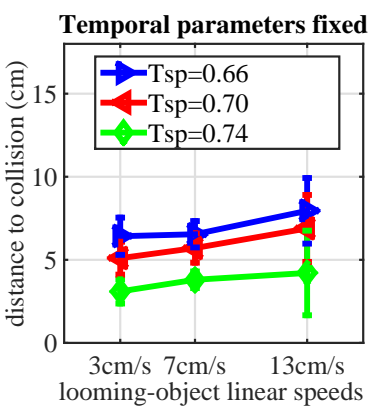

(a)

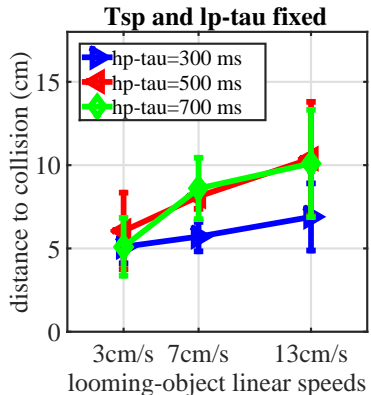

(b)

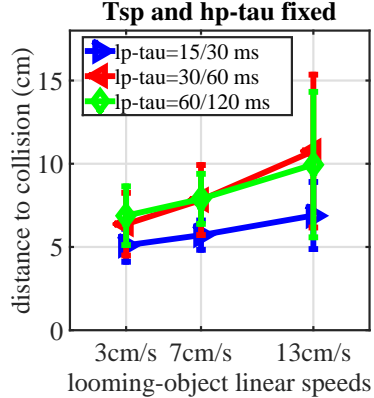

(c)

Figure 15: Statistical results (error bars) of DTC tests on different combinations of investigated parameters: the Colias robot approached an identical dark object at three linear-speeds. Each combination of parameters was repeated ten times at each speed. (a) The spiking threshold varies. (b) The temporal parameter in the SFA mechanism varies. (c) The temporal parameters in the $\mathrm{ON}$ and $\mathrm{OFF}$ pathways vary. The results demonstrate the speed response of the proposed neuron model tested by all combinations of investigated parameters in collision detection tasks.

designed experiments to test the proposed framework with various combinations of model parameters, including the spiking threshold, the temporal parameters in the low-pass filtering of the ON and OFF pathways and the high-pass filtering of the SFA mechanism. More specifically, the spiking threshold plays a crucial role in mediating the spike frequency of the proposed model. Compared with the comparative LGMD1 model, we apply a strategy of exponentially mapping the membrane potential to the firing rate. Moreover, instead of the 'one-framedelay' strategy in the comparative model, we compute the delayed signals by linear and temporal filtering the original signals with these investigated time parameters.

In the first round of DTC experiments, the Colias robot with the proposed model was used to approach an identical dark object at three constant linearspeeds. As illustrated in Fig. 15, the statistical results demonstrate the parameters all influence the DTC's response when tested at all speeds. The faster approaching speed gives rise to higher DTC response with each combination of parameters, underlying the speed response of the proposed looming detector. 

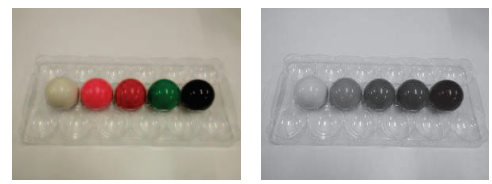

(a)

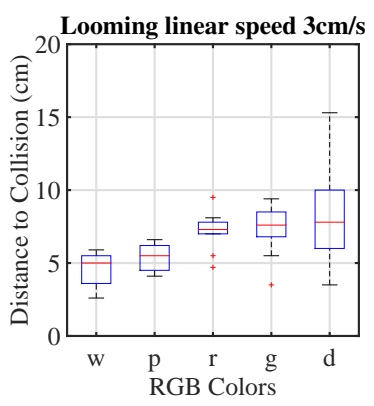

(b)

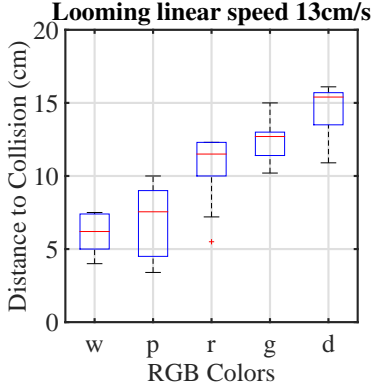

(c)

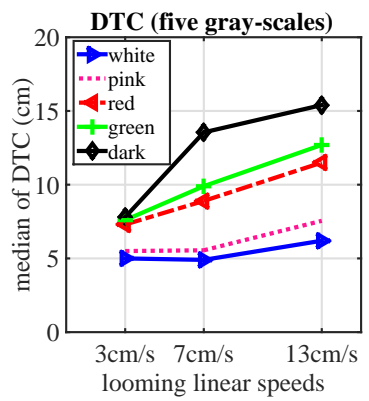

(d)

Figure 16: Statistical results of DTC tests on different grey-scaled looming stimuli: the Colias robot approached five grey-scaled obstacles (a) under the same parameters set at three linear speeds, respectively. Each test repeated ten times. (b) - (c) The box plots of DTC results at a slow and a fast linear speed, respectively. (d) The medians of DTC results. The embedded LGMD1 neuron model demonstrates both speed response and contrast sensitivity to looming stimuli.

More concretely, as shown in Fig. 15a, reducing the spiking threshold gives rise to larger DTC at all tested speeds. At each tested speed, the DTC increases by the shrinking of the spiking threshold. The results are in accordance with the computational rule of Eq. 21. that is, lower spiking threshold corresponds to higher firing rate with other parameters fixed.

On the aspect of temporal parameters in the proposed framework, Fig. 15b and $15 \mathrm{c}$ illustrate that these time parameters all have an influence on the speed response of the proposed neuron model. It appears that the DTC response at each tested speed peaks by a combination of temporal parameters of $500 \mathrm{~ms}$ for the high-pass filtering and 30/60 ms for the low-pass filtering. The results are also consistent with the spatiotemporal computations in the proposed framework: for temporal filtering in the polarity pathways, the delayed information decays more slowly by raising the time parameters (Eq. 4. 7), corresponding 
to stronger feedforward excitation and higher firing rate with other parameters

Angular approach tests. In the angular approach tests, we investigated the effects of direct looming as well as 'near-miss' scenes by the approaching stimulus from other angles. As illustrated in Fig. 17a, the motionless Colias robot was stimulated by an identical dark looming object from four distinct angles against which are also frequent challenges for visual collision detectors. We accumulated the elicited spikes from the proposed LGMD1 model during each angular 


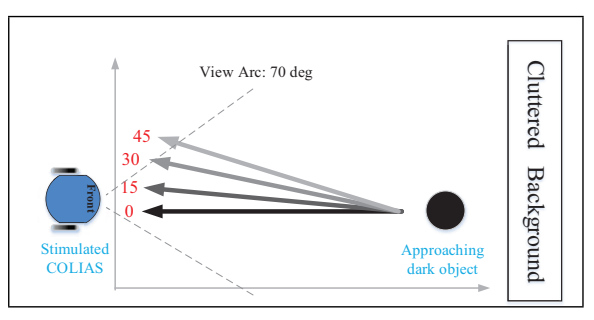

(a) Angular approaches

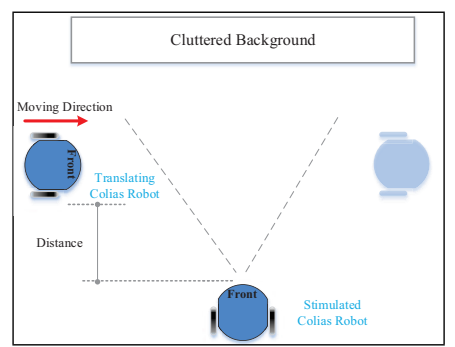

(b) Translations

Figure 17: Experimental settings of the angular approach and translation tests. In the angular approach tests, the stimulated Colias robot was motionless and challenged by a same dark object approaching from different angles against a cluttered background. In the translation tests, the stimulated Colias robot was challenged by translations of a moving robot.

approach with a course of approximately the same length of time throughout repeated tests. The results in Fig. 18 clearly demonstrate that the spike frequency (firing rate) of LGMD1 peaks when directly approaching. The spike rate declines gradually along with the increase of approaching angles. When stimulated by looming from 45 degrees, the largest angle tested, the LGMD1 model represented the lowest spike frequency. Our results verify that the proposed neuron model possesses similar characteristics to a biological LGMD1 neuron in locusts, which responds most strongly to directly approaching objects that represent the most powerful strikes from predators.

Translation tests. In the last part of the open-loop robot experiments, the $\mathrm{Co}$ lias robot was challenged by translations against a cluttered background, as illustrated in Fig. 17b. We investigated effects of the translation speed of visual stimulus and the distance from the stimulated robot. More specifically, the linear speed of the translating robot (stimulus) was set at approximately 3, 5, 7,10 and $13 \mathrm{~cm} / \mathrm{s}$, whilst the distance varied from 10 to $50 \mathrm{~cm}$.

As illustrated in Fig. 19, the statistical results demonstrate that both the 585 translating speed and the distance to the stimuli affect the peak neural response of the proposed LGMD1 neuron model. Concretely, the embedded LGMD1 neuron model represents the response speed to translation movements despite 


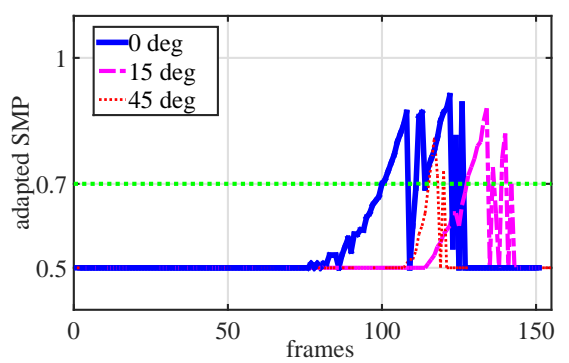

(a)

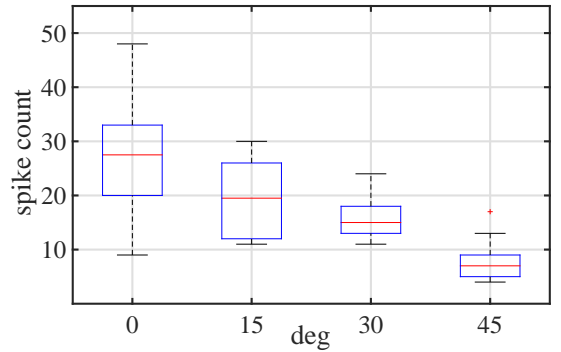

(b)

Figure 18: Angular approach tests results: (a) neural response of the embedded LGMD1 neuron model tested by three different angular approaches, (b) statistical results of the spike count (firing frequency) with each angle of looming tested by ten times. The spike frequency peaks at the direct looming, and declines as the increase of looming angle.

translations from the tested distance of $50 \mathrm{~cm}$. These results are consistent with the synthetic tests in Fig. 9. The faster translating speed gives rise to the stronger neural response of the proposed model. In addition, the nearby translations from a distance of $10 \mathrm{~cm}$ or closer also highly activate the proposed LGMD1 neuron model. It is conceivable that the locusts also treat the nearby fast translating objects as potential collision or dangers. In addition, if the translating distance is very far from the field of view ( $40 \sim 50 \mathrm{~cm}$ or further), the proposed LGMD1 model remained quiet for all tested translating speeds. In this case, the translating object corresponds to a small target, which may be identified by other kinds of visual neurons like the small target movement detectors [45, rather than the LGMDs.

\section{Discussion}

Through systematic experiments ranging from off-line to on-line tests, we have shown that the proposed looming sensitive neuron model, with the parallel polarity pathways and the spike frequency adaptation mechanism, demonstrates similar characteristics to the biological LGMD1 neuron and model (Fig. 5). More importantly, compared with a similar LGMD1 model from previous 


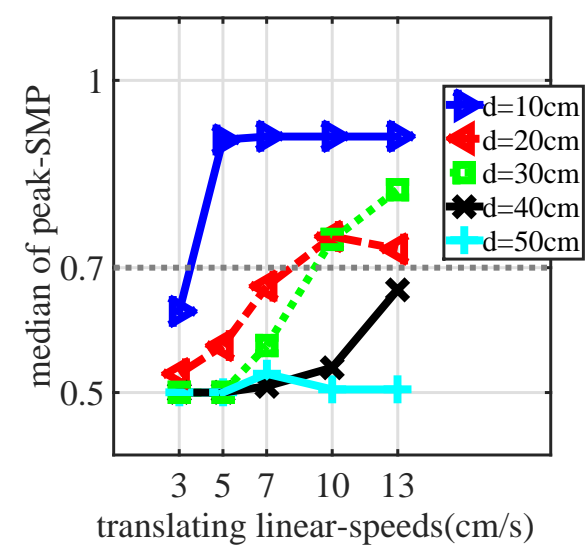

(a)

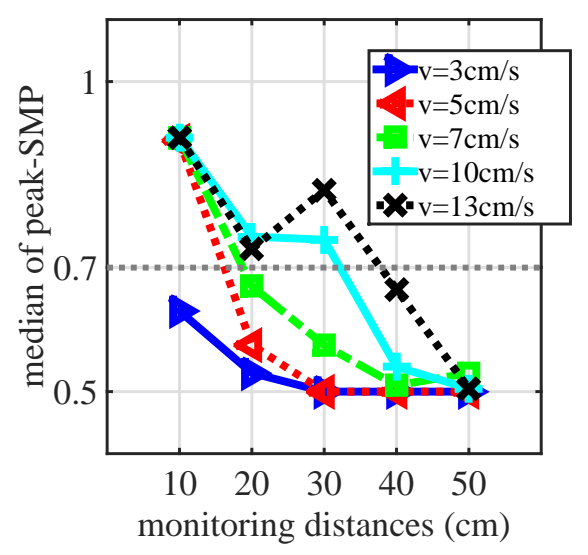

(b)

Figure 19: Statistical results of systematic translation experiments: each speed or distance was tested for ten times, respectively. The horizontal dashed-lines indicate the spiking threshold.

research that deals with visual processing in a single pathway [15, 27, we have demonstrated the efficacy of ON and OFF pathways in building a looming sensitive neural system. The collision selectivity is further enhanced for looming objects over other regular visual challenges, which have been exhibited by the above experiments. Unlike other animals such as the flies, the biological ON and OFF pathways have not yet been anatomically and physiologically identified in locusts, leaving little evidence to the computational modellers. However, this modelling study evidences that such ON and OFF mechanisms or pathways may exist locationally between the preliminary photoreceptors and the LGMDs.

Despite the ON and OFF pathways, another important achievement of this research is to demonstrate the efficacy of an SFA mechanism in shaping the collision selectivity. However, there is valuable data on different protocols for shaping the collision selectivity in LGMD1 that has not been compared thoroughly in this work. For example, a seminal work of a non-linear LGMD1 neuron model has demonstrated a non-linear correlation between the feedforward inhibitory and excitatory responses [16]. The FFI mechanism could also contribute effectively in mediating the collision selectivity of an LGMD1 neuron, which cannot 
be disregarded. We would like to compare the different mechanisms and investigate the collaboration of them in shaping the LGMD1's collision selectivity in our future work.

The arena tests have verified the effectiveness and robustness of the proposed neuron model for guiding collision avoidance in robotic navigation (Table 3. The comparative arena tests have also demonstrated the improved performance of collision detection of the proposed model over the comparative model (Table 4). Again, the efficacy of ON and OFF pathways for looming perception has been validated by the comparative experiments. We would like to further investigate the efficacy of this embedded collision-detecting vision system for collision detection in dynamic scenes mixed with multiple robots.

\section{Conclusions}

In this paper, we have presented a looming sensitive visual neural network based on a biological LGMD1 neuron in the locust's visual system. Although many LGMD-based modelling works have been successfully utilised for fast collision detection, shaping the collision selectivity to looming stimuli over other types of visual challenges is still an open challenge. In this modelling study, we have demonstrated the efficacy of biologically plausible ON and OFF pathways and spike frequency adaptation mechanism for looming perception and enhancing the LGMD1's collision selectivity. Our systematic experiments have verified that the collision selectivity of the proposed neuron model has been effectively enhanced to looming rather than translating and receding stimuli. The experiments have also shown the potential of ON and OFF pathways in achieving different collision selectivity among looming detectors, like the realisation of a biological LGMD2 neuron which is only sensitive to dark looming objects. Moreover, we have exhibited the role of spike frequency adaptation as a novel protocol in the proposed LGMD1 neuron model for shaping the collision selectivity.

The proposed LGMD1 model perceives looming via low-level spatiotempo- 
ral computation dealing with cluttered environments without applying complex image segmentation and object recognition strategies. The experiments have shown the significant potential to build neuromorphic sensors in the applications of robots and vehicles. Similar to other neuromorphic solutions, the proposed work can also be easily realised in VLSI chip for volume production.

In our future work, we will investigate the possibility of integrating direction and collision selective neural models with similar separated ON and OFF pathways to handle more complex navigating scenarios of robots and vehicles.

\section{Acknowledgment}

This work was supported by the grants of EU FP7 projects LIVCODE (295151), HAZCEPT (318907) and EU Horizon 2020 project STEP2DYNA (691154). We thank Mr. Tian Liu and Mr. Xuelong Sun for the Colias robot set-up. We thank Dr. Tomáš Krajník and Mr. Peter Lightbody for the help on robot localization system set-up in arena tests. We also thank Mr. Peter

Lightbody for language revision and proofreading of this paper.

\section{References}

[1] A. Mukhtar, L. Xia, T. B. Tang, Vehicle detection techniques for collision avoidance systems: A review, IEEE Transactions on Intelligent Transportation Systems 16 (5) (2015) 2318-2338. doi:10.1109/TITS.2015.2409109.

[2] G. N. DeSouza, A. C. Kak, Vision for mobile robot navigation: A survey, IEEE Transactions on Pattern Analysis and Machine Intelligence 24 (2002) 237-267.

[3] H. D., H. S., R. R.B., B. S., Real-time plane segmentation using rgb-d cameras, in: RoboCup 2011: Robot Soccer World Cup XV, Vol. 7416, Springer, 2012. 
[4] B. Peasley, S. Birchfield, Real-time obstacle detection and avoidance in the presence of specular surfaces using an active $3 \mathrm{~d}$ sensor, in: Robot Vision (WORV), 2013 IEEE Workshop on, 2013. doi:10.1109/WORV.2013. 6521938

[5] B. Schmidt, L. Wang, Depth camera based collision avoidance via active robot control, Journal of Manufacturing Systems 33 (4) (2014) 711-718.

[6] H. Kim, S. Leutenegger, A. J. Davison, Real-time 3d reconstruction and 6dof tracking with an event camera, in: European Conference on Computer Vision, 2016, pp. 1-16.

[7] J. R. Serres, F. Ruffier, Optic flow-based collision-free strategies: From insects to robots, Arthropod Structure \& Development 46 (5) (2017) 703717.

[8] G. Indiveri, R. Douglas, Neuromorphic vision sensors, Science (2000) 11891190.

[9] Q. Fu, C. Hu, T. Liu, S. Yue, Collision selective neuron models research benefit from a vision-based autonomous micro robot, in: 2017 IEEE/RSJ International Conference on Intelligent Robots and Systems (IROS), IEEE, 2017.

[10] F. C. Rind, B. D. I., Neural network based on the input organization of an identified neurone signaling impending collision, J Neurophysiol 75 (1996) 967-985.

[11] P. J. Simmons, F. C. Rind, Responses to object approach by a wide field visual neurone, the lgmd2 of the locust: Characterization and image cues, J Comp Physiol A 180 (1997) 203-214.

[12] F. Gabbiani, H. G. Krapp, N. Hatsopoulos, C. H. Mo, C. Koch, G. Laurent, Multiplication and stimulus invariance in a looming-sensitive neuron, $\mathrm{J}$ Physiol Paris 98 (1-3) (2004) 19-34. 
[13] F. C. Rind, S. Wernitznig, P. Polt, A. Zankel, D. Gutl, J. Sztarker, G. Leitinger, Two identified looming detectors in the locust: ubiquitous lateral connections among their inputs contribute to selective responses to looming objects, Scientific Reportsdoi:10.1038/srep35525.

[14] H. Fotowat, A. Fayyazuddin, H. J. Bellen, F. Gabbiani, A novel neuronal pathway for visually guided escape in drosophila melanogaster, J Neurophysiol 102 (2) (2009) 875-885.

[15] C. Hu, F. Arvin, C. Xiong, S. Yue, Bio-inspired embedded vision system for autonomous micro-robots: The lgmd case, IEEE Transactions on Cognitive and Developmental Systems 9 (3) (2017) 241-254.

[16] S. Bermudez i Badia, U. Bernardet, P. F. Verschure, Non-linear neuronal responses as an emergent property of afferent networks: a case study of the locust lobula giant movement detector, PLoS Comput Biol 6 (3) (2010) e1000701.

[17] S. Yue, F. C. Rind, A collision detection system for a mobile robot inspired by locust visual system, in: Proc. IEEE Int. Conf. Robot. Autom., 2005, pp. $3843-3848$.

[18] S. Yue, R. D. Santer, Y. Yamawaki, F. C. Rind, Reactive direction control for a mobile robot: a locust-like control ofescape direction emerges when abilateral pair of model locust visual neurons are integrated, Autonomous Robots 28 (2) (2010) 151-167.

[19] S. Yue, F. C. Rind, Visually stimulated motor control for a robot with a pair of lgmd visual neural networks, Int. J. Adv. Mechatron. Syst. 4 (5) (2012) 237-247.

[20] Q. Fu, S. Yue, Modelling lgmd2 visual neuron system, in: 2015 IEEE 25th International Workshop on Machine Learning for Signal Processing. 
[21] Q. Fu, C. Hu, S. Yue, A bio-inspired collision detector with enhanced selectivity for ground robotic vision system, in: British Machine Vision Conference 2016, 2016.

[22] M. Hartbauer, Simplified bionic solutions: a simple bio-inspired vehicle collision detection system, Bioinspiration and Biomimetics 12 (2).

[23] S. Yue, F. C. Rind, M. S. Keil, J. Cuadri, R. Stafford, A bio-inspired visual collision detection mechanism for cars: Optimisation of a model of a locust neuron to a novel environment, Neurocomputing 69 (13-15) (2006) 15911598.

[24] R. Stafford, R. D. Santer, F. C. Rind, A bio-inspired visual collision detection mechanism for cars: combining insect inspired neurons to create a robust system, Biosystems 87 (2-3) (2007) 164-71.

[25] S. Yue, F. Claire Rind, Visual motion pattern extraction and fusion for collision detection in complex dynamic scenes, Computer Vision and Image Understanding 104 (1) (2006) 48-60.

[26] W. E. Green, P. Y. Oh, Optic-flow-based collision avoidance, IEEE Robotics Automation Magazine 15 (1) (2008) 96-103.

[27] S. Yue, F. C. Rind, Collision detection in complex dynamic scenes using a lgmd based visual neural network with feature enhancement, IEEE Trans. Neural Netw. 17 (3) (2006) 705-716.

[28] F. Gabbiani, P. W. Jones, A genetic push to understand motion detection, Neuron 70 (6) (2011) 1023-5.

[29] J. Rister, D. Pauls, B. Schnell, C.-Y. Ting, C.-H. Lee, I. Sinakevitch, J. Morante, N. J. Strausfeld, K. Ito, M. Heisenberg, Dissection of the peripheral motion channel in the visual system of drosophila melanogaster, Neuron 56 (1) (2007) 155-170. 
[30] M. Joesch, B. Schnell, S. V. Raghu, D. F. Reiff, A. Borst, On and off pathways in drosophila motion vision, Nature 468 (7321) (2010) 300-304.

[31] A. Borst, Fly visual course control: behaviour, algorithms and circuits, Nature Reviews Neuroscience 15 (2014) 590-599.

[32] A. Leonhardt, G. Ammer, M. Meier, E. Serbe, A. Bahl, A. Borst, Asymmetry of drosophila on and off motion detectors enhances real-world velocity estimation, Nature Neuroscience 19 (2016) 706-715.

[33] K. Shinomiya, S. ya Takemura, P. K. Rivlin, S. M. Plaza, L. . Scheffer, I. A. Meinertzhagen, A common evolutionary origin for the on- and offedge motion detection pathways of the drosophila visual system, Frontiers in Neural Circuits 9 (33) (2015) 00033.

[34] J. Antolik, Rapid long-range disynaptic inhibition explains the formation of cortical orientation maps, Frontiers in Neural Circuits 11 (21) (2017) 00021 .

[35] A. Borst, T. Euler, Seeing things in motion: models, circuits, and mechanisms, Neuron 71 (6) (2011) 974-94.

[36] A. Borst, M. Helmstaedter, Common circuit design in fly and mammalian motion vision, nature neuroscience 18 (2015) 1067-1076.

[37] T. W. Troyer, A. E. Krukowski, N. J. Priebe, K. D. Miller, Contrastinvariant orientation tuning in cat visual cortex: Thalamocortical input tuning and correlation-based intracortical connectivity, The Journal of Neuroscience 18 (15) (1998) 5908-5927.

[38] L. Chariker, R. Shapley, L.-S. Young, Orientation selectivity from very sparse lgn inputs in a comprehensive model of macaque v1 cortex, The Journal of Neuroscience 36 (49) (2016) 12368-12384.

[39] D. A. Clark, L. Bursztyn, M. A. Horowitz, M. J. Schnitzer, T. R. Clandinin, Defining the computational structure of the motion detector in drosophila, Neuron 70 (6) (2011) 1165-1177. 
[40] M. Joesch, F. Weber, H. Eichner, A. Borst, Functional specialization of parallel motion detection circuits in the fly, J Neurosci 33 (3) (2013) 902905.

[41] H. Eichner, M. Joesch, B. Schnell, D. F. Reiff, A. Borst, Internal structure of the fly elementary motion detector, Neuron 70 (6) (2011) 1155-64.

[42] S. D. Wiederman, P. A. Shoemaker, D. C. O'Carroll, Correlation between off and on channels underlies dark target selectivity in an insect visual system, J Neurosci 33 (32) (2013) 13225-32.

[43] Q. Fu, S. Yue, Modeling direction selective visual neural network with on and off pathways for extracting motion cues from cluttered background, in: The 2017 International Joint Conference on Neural Networks, 2017.

[44] H. Wang, J. Peng, S. Yue, Bio-inspired small target motion detector with a new lateral inhibition mechanism, in: The 2016 International Joint Conference on Neural Networks, 2016.

[45] S. D. Wiederman, P. A. Shoemaker, D. C. O'Carroll, A model for the detection of moving targets in visual clutter inspired by insect physiology, PLoS ONE 3 (7) (2008) e2784. doi:10.1371/journal.pone.0002784.

${ }_{800}$ [46] S. Peron, F. Gabbiani, Spike frequency adaptation mediates looming stimulus selectivity in a collision-detecting neuron, Nat Neurosci 12 (3) (2009) $318-26$.

[47] S. P. Peron, F. Gabbiani, Role of spike-frequency adaptation in shaping neuronal response to dynamic stimuli, Biol Cybern 100 (6) (2009) 505-20.

[48] L. F. Tammero, M. H. Dickinson, Collision-avoidance and landing responses are mediated by separate pathways in the fruit fly, drosophila melanogaster, The Journal of Experimental Biology 205 (2002) 2785-2798.

[49] G. Maimon, A. D. Straw, M. H. Dickinson, A simple vision-based algorithm for decision making in flying drosophila, Current Biology 18 (6) (2008) 464470. 
[50] A. Borst, J. Haag, D. F. Reiff, Fly motion vision, The Annual Review of Neuroscience 33 (2010) 49-70.

[51] O. J. N. Bertrand, J. P. Lindemann, M. Egelhaaf, A bio-inspired collision avoidance model based on spatial information derived from motion detectors leads to common routes, PLOS Computational Biology 11 (11) (2015) $1-28$.

[52] F. Poiesi, A. Cavallaro, Bioinspired event-driven collision avoidance algorithm based on optic flow, in: British Machine Vision Conference, 2016, pp. 1-11.

[53] M. B. Milde, O. J. N. Bertrand, R. Benosmanz, M. Egelhaaf, E. Chicca, Bioinspired event-driven collision avoidance algorithm based on optic flow, in: 2015 International Conference on Event-based Control, Communica-

a tion, and Signal Processing (EBCCSP), 2015, pp. 1-7. doi:10.1109/ EBCCSP. 2015.7300673,

${ }_{825}$ [54] P. J. Simmons, F. C. Rind, R. D. Santer, Escapes with and without preparation: the neuroethology of visual startle in locusts, J Insect Physiol 56 (8) (2010) 876-83.

[55] J. Sztarker, F. C. Rind, A look into the cockpit of the developing locust: looming detectors and predator avoidance, Dev Neurobiol 74 (11) (2014) 1078-95.

[56] C. Hu, F. Arvin, S. Yue, Development of a bio-inspired vision system for mobile micro-robots, in: Development and Learning and Epigenetic Robotics (ICDL-Epirob), IEEE, 2014, pp. 81-86.

[57] M. Hongying, Y. Shigang, H. Andrew, A. Kofi, H. Mervyn, P. Nigel, H. Peter, P. Cy, A modified neural network model for lobula giant movement detector with additional depth movement feature, in: International Joint Conference on Nerual Networks 2009, IEEE, 2009, pp. 2078-2083. 
[58] M. O'Shea, C. H. F. Rowell, The neuronal basis of a sensory analyser, the acridid movement detector system. ii. response decrement, convergence, and the nature of the excitatory afferents to the fan-like dendrites of the lgmd, J Exp Biol 65 (1976) 289-308.

[59] S. Wernitznig, F. C. Rind, P. Polt, A. Zankel, E. Pritz, D. Kolb, E. Bock, G. Leitinger, Synaptic connections of first-stage visual neurons in the locust schistocerca gregaria extend evolution of tetrad synapses back 200 million years, J Comp Neurol 523 (2) (2015) 298-312.

[60] M. S. Keil, E. Roca-Moreno, A. Rodriguez-Vazquez, A neural model of the locust visual system for detection of object approaches with real-world scenes, in: Proceedings of the Fourth IASTED, 2004, pp. 340-345.

[61] F. Gabbiani, H. G. Krapp, Spike-frequency adaptation and intrinsic properties of an identified, looming-sensitive neuron, J Neurophysiol 96 (6) (2006) 2951-62.

[62] A. Borst, M. Egelhaaf, Principles of visual motion detection, Trends Neurosci 12 (1989) 297-306.

[63] S. Yue, F. C. Rind, A synthetic vision system using directional selective motion detectors for collision recognition, Artificial Life 13 (2) (2007) 93122.

[64] S. Yue, F. C. Rind, Postsynaptic organization of directional selective visual neural networks for collision detection, Neurocomput 103 (2013) 50-62.

[65] S. Yue, F. C. Rind, Redundant neural vision systemscompeting for collision recognition roles, IEEE Transactions on Autonomous Mental Development 5 (2) (2013) 173-186.

[66] F. Arvin, J. Murray, C. Zhang, S. Yue, Colias: An autonomous micro robot for swarm robotic applications, International Journal of Advanced Robotic Systems (2014) 1-10. 
[67] F. Arvin, T. Krajnik, A. E. Turgut, S. Yue, Cos: Artificial pheromone system for robotic swarms research, in: 2015 IEEE/RSJ International Conference on Intelligent Robots and Systems (IROS), IEEE, 2015, pp. 407-412.

[68] T. Krajník, M. Nitsche, I. Faigl, P. Vaněk, M. Saska, L. Přeučil, T. Duckett, M. Marta, A practical multirobot localization system, Journal of Intelligent and Robotic Systems 76 (3-4) (2014) 539-562.

[69] P. Lightbody, M. Hanheide, T. Krajník, An efficient visual fiducial localisation system, Applied Computing Review 17 (3) (2017) 28-37.

[70] Peter Lightbody and Marc Hanheide and Tomáš Krajník, A versatile highperformance visual fiducial marker detection system with scalable identity 875 encoding, in: 32nd ACM Symposium on Applied Computing, 2017. 